\title{
Supplementary Motor Area Exerts Proactive and Reactive Control of Arm Movements
}

\author{
Xiaomo Chen, ${ }^{1,3}$ Katherine Wilson Scangos, ${ }^{2,3}$ and Veit Stuphorn ${ }^{1,2,3}$ \\ ${ }^{1}$ Department of Psychological and Brain Sciences, Johns Hopkins University, Baltimore, Maryland 21218, ${ }^{2}$ Department of Neuroscience, Johns Hopkins \\ University School of Medicine, Baltimore, Maryland 21205, and ${ }^{3}$ Zanvyl Krieger Mind/Brain Institute, Baltimore, Maryland 21218
}

\begin{abstract}
Adaptive behavior requires the ability to flexibly control actions. This can occur either proactively to anticipate task requirements, or reactively in response to sudden changes. Here we report neuronal activity in the supplementary motor area (SMA) that is correlated with both forms of behavioral control. Single-unit and multiunit activity and intracranial local field potentials (LFPs) were recorded in macaque monkeys during a stop-signal task, which elicits both proactive and reactive behavioral control. The LFP power in high- $(60-150$ $\mathrm{Hz}$ ) and low- (25-40 Hz) frequency bands was significantly correlated with arm movement reaction time, starting before target onset. Multiunit and single-unit activity also showed a significant regression with reaction time. In addition, LFPs and multiunit and single-unit activity changed their activity level depending on the trial history, mirroring adjustments on the behavioral level. Together, these findings indicate that neuronal activity in the SMA exerts proactive control of arm movements by adjusting the level of motor readiness. On trials when the monkeys successfully canceled arm movements in response to an unforeseen stop signal, the LFP power, particularly in a low $(10-50 \mathrm{~Hz})$ frequency range, increased early enough to be causally related to the inhibition of the arm movement on those trials. This indicated that neuronal activity in the SMA is also involved in response inhibition in reaction to sudden task changes. Our findings indicate, therefore, that SMA plays a role in the proactive control of motor readiness and the reactive inhibition of unwanted movements.
\end{abstract}

\section{Introduction}

Voluntary control entails the ability to flexibly adapt behavior to a changing environment and can take at least two different forms (Braver et al., 2007). First, reactive control is a transient process, triggered by an unexpected change in the task requirements that cancels or modifies the response preparation. Second, proactive control adjusts the response selection and preparation process in anticipation of known task demands. Proactive control is guided by endogenous signals, instead of external triggers, and is constantly present throughout response selection and preparation. It can reflect a variety of factors, such as the incentives for choosing different responses and the frequency of task-relevant events.

The stop-signal (or countermanding) task evokes both reactive and proactive forms of control. Subjects performing this task are required to cancel a response at variable stages of preparation when presented with an infrequent stop signal. This inhibitory process represents a form of reactive control and has been the focus of most physiological studies in the oculomotor (Hanes et al., 1998; Stuphorn et al., 2000; Ito et al., 2003; Paré and Hanes, 2003) and skeletomotor (Scangos and Stuphorn, 2010) system. However, task performance is also influenced by factors that are independent of the presence of an actual stop signal (Verbruggen and Logan, 2009). Behavioral studies in monkeys and humans

\footnotetext{
Received May 26, 2010; revised Aug. 31, 2010; accepted Sept. 2, 2010.

This work was supported by the National Eye Institute through Grant R01-EY019039 to V.S. We are grateful to J. Brown, E. Emeric, and J. D. Schall for comments on this manuscript.

Correspondence should be addressed to Veit Stuphorn, Johns Hopkins University, 338 Krieger Hall, 3400 North Charles Street, Baltimore, MD 21218. E-mail: veit@jhu.edu.

DOI:10.1523/JNEUROSCI.2669-10.2010

Copyright $\odot 2010$ the authors $\quad 0270-6474 / 10 / 3014657-19 \$ 15.00 / 0$
}

show that the mean response time during no-stop-signal trials is delayed relative to a situation when no stop signal is expected (Verbruggen et al., 2004, 2006; Stuphorn and Schall, 2006). Short-term changes in stop-signal frequency lead to behavioral adjustments (Emeric et al., 2007). These systematic modulations in the mean reaction time indicate the presence of proactive control. We hypothesize that by adjusting the level of excitation and inhibition of the motor system, the proactive control system sets the threshold for initiating a response. In making these adjustments, the proactive system has to negotiate the tradeoff between speed (reaction time) and accuracy (cancelation likelihood) (Bogacz et al., 2010).

A network of brain areas in the frontal cortex and the basal ganglia underlies behavioral control (Floden and Stuss, 2006; Aron et al., 2007b; Picton et al., 2007). A critical component of this network is the medial frontal cortex, in particular the presupplementary motor area (pre-SMA) and adjacent SMA. PreSMA and SMA differ in their connection pattern, with pre-SMA exclusively connected to prefrontal cortex, and SMA to motor regions (Johansen-Berg et al., 2004). Recent neuroimaging studies of humans in the countermanding task show activity centered on the pre-SMA (Curtis et al., 2005; Aron and Poldrack, 2006; Li et al., 2006; Aron et al., 2007a). However, lesion studies indicate an important role of SMA in inhibition, as well (Sumner et al., 2007). To clarify the role of SMA in behavioral control, we recorded local field potentials (LFPs), multiunit activity, and single-unit activity from this area in macaque monkeys performing a stop-signal task. Our results clearly indicate that SMA contributes both to proactive and reactive control of arm movements. 


\section{Materials and Methods}

General. Two rhesus monkeys (B, male, $8.5 \mathrm{~kg}$; and $\mathrm{E}$, female, $6.8 \mathrm{~kg}$ ) were trained to perform the tasks used in this study. All animal care and experimental procedures were approved by Johns Hopkins University Animal Care and Use Committee. During the experimental sessions, each monkey was seated in a primate chair, with its head restrained, facing a video screen. A handlebar was placed in front of the monkey that moved $12 \mathrm{~cm}$ in either direction along the horizontal axis. The bar controlled a rectangular cursor on the video screen. The right (contralateral) arm was used for the task. Handlebar position was recorded with the PLEXON system (Plexon) at a sampling rate of $1000 \mathrm{~Hz}$. Eye movement was monitored with an infrared corneal reflection system (SR Research) at a sampling rate of $1000 \mathrm{~Hz}$. On a subset of experimental sessions, electromyographic (EMG) activity was recorded using surface electrodes on six different muscles (pectoralis, deltoid, biceps, triceps, flexor carpi radialis, and extensor carpi radialis).

Behavioral tasks. The stop-signal (or countermanding) task provided the main experimental data for this report. Each trial began with the onset of a yellow center box presented centrally on the monitor (Fig. 1), instructing the monkey to move the cursor into the box. Following a variable delay $(200-400 \mathrm{~ms})$, the center box disappeared and a target box simultaneous appeared 16 visual degrees to the right (contralateral) or left (ipsilateral) of center. On no-stop-signal trials, the monkey was reinforced by a liquid reward for moving the cursor into the target box within $700 \mathrm{~ms}$. On $25-35 \%$ of trials, a stop signal was presented. On these stop-signal trials, the center box reappeared after a delay, referred to as the stop-signal delay (SSD), and the monkey was required to withhold movement initiation to receive reward. The reward was delivered 400-800 ms after the stop-signal onset. In contrast to previously reported countermanding paradigms, the monkey was reinforced for correcting movements toward the target, if it was able to interrupt this movement before the target was reached and returned the cursor back to the center. The intertrial interval was in all cases 1000 ms. Four stop-signal delays ranging from 120 to 420 ms were selected through baseline training sessions so the monkey could withhold movement on $\sim 10,35,65$, and $90 \%$ of the stop trials. In the variable reward version of the stop-signal task, the color of the initial center box (green vs yellow) signaled whether a correct trial would result in a high or low reward. The high reward was always twice as large as the low reward. High-reward trials were presented at random on $25-30 \%$ of trials. The variable-reward version and the fixed version of the task were performed in separate sessions. The SSD levels were the same for both tasks.

Behavioral analysis. The relevant behavioral data for describing the inhibitory process in the stop-signal task are (1) the inhibition function and (2) the reaction time distribution for the movements on trials with no stop signals. The inhibition function plots the proportion of stopsignal trials in which the subject generates a movement as a function of the delay between target onset and stop-signal onset (the stop-signal delay). The probability of erroneous initiation of the movement increases as stop-signal delay increases. Performance on the countermanding task can be modeled as a race between a stochastic process that generates the movement (GO process), and a stochastic process that inhibits the movement (STOP process) (Logan and Cowan, 1984). The two processes race independently toward their respective thresholds. If the STOP process finishes before the GO process, the movement is not generated (canceled trials). On the other hand, if the GO process finishes before the STOP process, the movement is generated (noncanceled trials). This race model provides an estimate of the stop-signal reaction time (SSRT), which is the time needed to cancel the planned movement.

We estimated the SSRT using methods detailed previously (Hanes and Schall, 1995; Hanes et al., 1998). First, we used the method of integration, based on the assumption that SSRT is constant. This method provided an estimate of the SSRT at each SSD by integrating the go trial reaction time distribution, until the integral equaled the proportion of noncanceled error trials at that SSD (Logan et al., 1984). The SSRT could then be obtained by subtracting the SSD from this value. We took the mean of the SSRTs calculated individually for each SSD.

The second method did not assume that the SSRT is a constant variable. This method asserted that the mean SSRT is equal to the difference between the mean reaction time during go trials and the mean value of the inhibition function. The mean of the inhibition function was found by converting it to a probability density function. The mean was then the difference between the probability of responding at the ith stop-signal delay and the probability of responding at the $(i-1)$ th SSD, multiplied by the $i$ th SSD, summed over all SSDs. The SSRT was then estimated by subtracting the mean of the inhibition function from the mean go trial reaction time.

Using two variants of this general "constant SSRT” approach, we fitted a Weibull function and a monotonic spline function to the inhibition function. These functions were then used to find the SSD at which the probability of making a movement is 0.5 . The SSRT was estimated by subtracting the median of the Weibull or spline function from the mean go trial reaction time. We obtained an overall estimate of SSRT by averaging over the four different estimates.

The difficulty of controlling the movement generation can be adjusted parametrically by changing the stop-signal delay. As a result, the monkey is fully engaged in the task, but still generates a similar number of noncanceled, corrected, and canceled stop-signal trials overall. The proportion of each type of stop trial will vary by SSD, however, which forms the basis of the inhibition function. In our calculations of the inhibition function, noncanceled and corrected trials are counted as incorrect, since the monkey incorrectly makes a movement in both of these trial types. 
A Monkey B
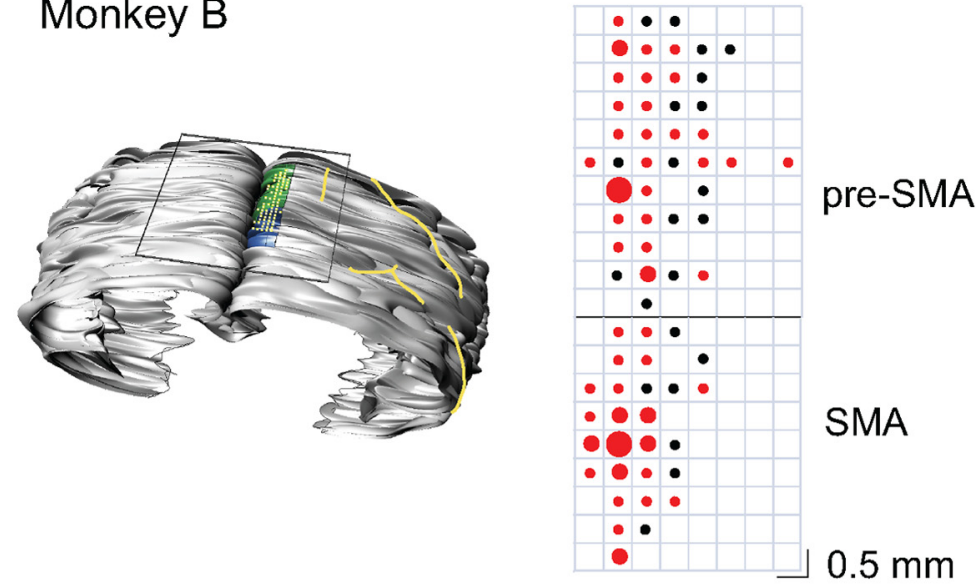

B

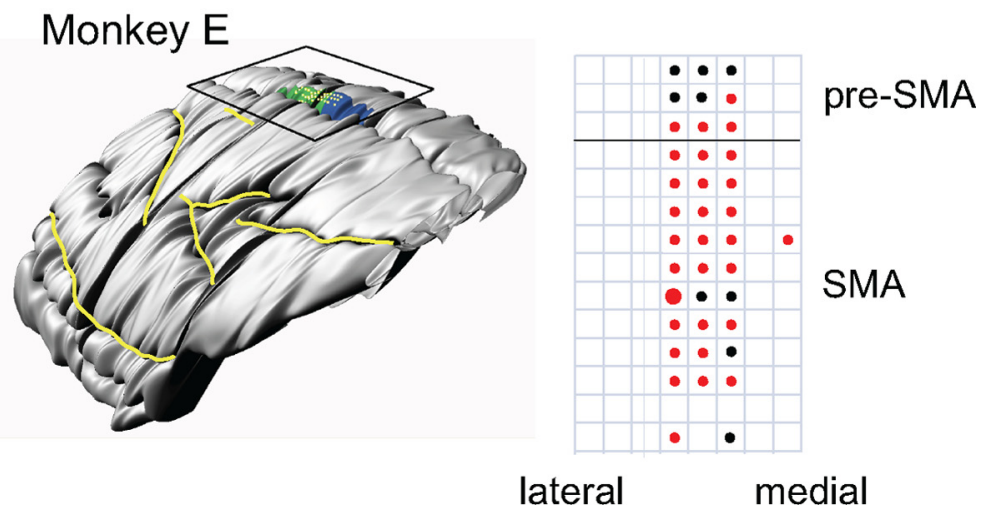

Figure 2. Localization of recording sites. A, A model of the brain for monkey B constructed from MRI slices showing the pre-SMA region highlighted in blue and the SMA region highlighted in green (left). Major sulci are outlined in yellow. The black boxindicates the location of the recording chamber and the yellow grid indicates the recording sites. A magnified version of the recording grid is shown to the right. The circles indicate the location of electrode penetrations. The red circle sizes indicate the number of movement-related neurons found at that location (large: 9-12 cells, medium: 5-8 cells, small: $1-4$ cells). Penetrations that yielded no movement-related neurons are indicated by black dots. The horizontal black line indicates the location of the branch of the arcuate sulcus. $\boldsymbol{B}$, Chamber location and recording sites from monkey $\mathrm{E}$.

Recording of local field potentials and single units. After training, we placed a square chamber $(20 \times 20 \mathrm{~mm})$ centered over the midline, 25 $\mathrm{mm}$ (monkey B) or $21 \mathrm{~mm}$ (monkey E) anterior of the interaural line. Neuronal activity was recorded using tungsten microelectrodes with an impedance of 2-4 M $\Omega$ s and collected using the PLEXON system. At the preamplifier stage, the electrophysiological recording signal was divided into two streams for the collection of LFPs and single-unit spikes, respectively. The stream used for LFP recording was amplified ( $50 \times$ gain), low-pass filtered $(0.7-170 \mathrm{~Hz})$ and collected at a sampling rate of 1000 $\mathrm{Hz}$. The stream used for spike identification was high-pass filtered (100 $\mathrm{Hz}$ to $8 \mathrm{kHz}$ ), up to four template spikes were identified using principal component analysis, and the time stamps were then collected at a sampling rate of $1000 \mathrm{~Hz}$. Data were subsequently analyzed offline to ensure the quality of single-unit identification.

Cortical localization. To determine the locations of the pre-SMA and SMA, we obtained magnetic resonance images (MRIs) for both monkeys $(1.5 \mathrm{~T})$. A three-dimensional model of the brain was constructed using Brain Voyager (Brain Innovation) and Rhinoceros (McNeel North America) (Fig. 2). The border of the SMA and pre-SMA was defined by the location of the branch of the arcuate sulcus. Neurons within the region $6 \mathrm{~mm}$ posterior to the arcuate branch and within $3.5 \mathrm{~mm}$ of the longitudinal fissure were designated as belonging to the SMA. Neurons within the region $5 \mathrm{~mm}$ anterior to the arcuate branch and within $3.5 \mathrm{~mm}$ of the longitudinal fissure were designated as belonging to the pre-SMA. The location of neuronal recording sites and the number of movementrelated neurons is shown to the right of Figure 2. In monkey B, note the two clusters of arm-movement-related neurons above and below the anterior-posterior level of the arcuate branch corresponding to pre-SMA and SMA, respectively (Fig. 2A).

Identification of arm and eye movements. The analog data from the handlebar was analyzed to find the beginning and end of arm movements. First, the position signal was smoothed by taking the average of every five data points. To determine movement onset, we first identified locations where there were five changes in handlebar position that were at most $25 \mathrm{~ms}$ away from one another. Movement onset was defined as the time at which the first of the five handlebar position changes occurred. We used this method instead of a velocity cutoff because it allowed us to examine the velocity without requiring a specific speed to be reached. We found that the method very consistently identified the beginning of movement. Movement end was defined as the first point after movement onset where the position stayed constant for $>40 \mathrm{~ms}$. The analog data from the eye was much noisier than that from the handlebar. Data were smoothed by taking the average of every 20 data points and the velocity was examined. First, position changes over $150 \mathrm{deg} / \mathrm{s}$ were identified. Movement onset and end were then determined by moving backward and forward in time from this maximum until a position change of $<5 \mathrm{deg} / \mathrm{s}$ was identified. We visually inspected the movement start and end times for eye and arm movements that were found using these algorithms to ensure their accuracy.

Spike density functions. To represent neural activity as a continuous function, we calculated spike density functions by convolving the spike train with a growth-decay exponential function that resembled a postsynaptic potential. Each spike therefore exerts influence only forward in time. The equation describes rate $(R)$ as a function of time $(t): R(t)=[1-\exp (-t)$ $\left.\left.\tau_{\mathrm{g}}\right)\right] \cdot \exp \left(-t / \tau_{\mathrm{d}}\right)$, where $\tau_{\mathrm{g}}$ is the time constant for the growth phase of the potential, and $\tau_{\mathrm{d}}$ is the time constant for the decay phase. Based on physiological data from excitatory synapses, we used $1 \mathrm{~ms}$ for the value of $\tau_{\mathrm{g}}$ and $20 \mathrm{~ms}$ for the value of $\tau_{\mathrm{d}}$ (Sayer et al., 1990).

Time-frequency analysis. Time-frequency analysis was performed using the matching pursuit (MP) algorithm (Mallat and Zhang, 1993). Matching pursuit has been used previously to analyze LFPs (Ray et al., 2008b; Swann et al., 2009). It is a procedure for computing adaptive signal representations and can eliminate all cross terms of the Wigner distribution of the signal. The Gabor function dictionaries used in MP provide the best time-frequency resolution possible in agreement with the uncertainty principle. The multiscale decomposition of MP allows sharp transients in the LFP signal to be represented by functions that have narrow temporal support, rather than oscillatory functions with a temporal support of hundreds of milliseconds (such as in short-time Fourier transform in time-frequency analysis/multitapering for spectrum analysis) (Ray et al., 2008a,b).

The algorithm is an iterative procedure that selects a set of Gabor functions (atoms) from a redundant dictionary of functions that constitute the best possible description of the original signal. Time-frequency plots were then obtained by calculating the Wigner distribution of every atom and taking the weighted sum. All computations were performed using MATLAB (MathWorks). We performed the MP computation using custom MATLAB scripts and the free software toolbox "LastWave" (http://www.cmap.polytechnique.fr/ bacry/LastWave/), developed by Emmanuel Bacry. 
The LFP signal was sampled at $1 \mathrm{kHz}$ and was analyzed in 900 separate $1 \mathrm{~ms}$ time bins. For stimulus-related activity, we examined the LFP signal in the $900 \mathrm{~ms}$ time interval starting $300 \mathrm{~ms}$ before target onset. For movement-related activity, we examined the $900 \mathrm{~ms}$ time interval starting $500 \mathrm{~ms}$ before movement onset. Matching pursuit yields a $430 \times 615$ array of time-frequency values (with a time resolution of $\sim 1.5 \mathrm{~ms}$, frequency resolution of $\sim 0.35 \mathrm{~Hz}$ ). For the regression analysis as well as between-group comparison, the arrays were further downsampled by a factor of 4 (the mean of every $4 \times 4$ pixels was taken as one timefrequency bin), yielding a $107 \times 153$ array with a time resolution of $6 \mathrm{~ms}$ and frequency resolution of $1.4 \mathrm{~Hz}$. For each array, we calculated the relative change in power (in decibel) by subtracting for each time-frequency bin on each trial the mean baseline activity in the period between $-550 \mathrm{~ms}$ and $-400 \mathrm{~ms}$ relative to target onset. The grand average was calculated across all the recording sites for each monkey in a certain experimental condition.

Relationship of neuronal activity and reaction time. A linear regression analysis was used to determine the relationship between time-frequency power and arm movement initiation time:

$$
\mathrm{RT}=b_{0}+b_{1} \times \operatorname{Ef}(t, \omega),
$$

where RT is the arm movement start time, $\operatorname{Ef}(t, \omega)$ is the power density of the LFP with $\Delta t$ and $\Delta \omega$ at time $t$ and frequency $\omega$. For each recording site, we tested the regression at every pixel within the map across a time period of $900 \mathrm{~ms}$ and a $0.7-150 \mathrm{~Hz}$ frequency band. We included only no-stop-signal trials in this analysis, since the inclusion of noncanceled stop-signal trials would have introduced a bias for shorter response times. The null hypothesis that time-frequency power of LFP in preSMA and SMA do not correlate with the reaction time of corresponding trials was evaluated using a $t$ test for each pixel in each recording site. Therefore, for each pixel, a $p$ value and coefficient value $b_{1}$ were calculated.

We applied a nonparametric cluster-based test originally suggested for use with EEG and MEG data (Maris and Oostenveld, 2007) to test the overall significance of the effects. This test is related to the "cluster mass" test used in the functional MRI literature (Bullmore et al., 1999). The advantage of the test is that it takes into account the additional information that comes from the temporal or spectral adjacency of pixels with significant regression slope values. We first selected all time-frequency pixels with $t$ values that were above the 97.5 th or below the 2.5 th percentile of the $t$ distribution. This threshold level was not used for the statistical test, but rather as a threshold for considering a pixel as a candidate member of some cluster. We clustered all pixels above (or below) the threshold with other above (or below)-threshold pixels of similar sign that were contiguous in either time or frequency. The $t$ values in each contiguous cluster were summed to determine the test statistic for each cluster. We then randomly shuffled the condition assignments between trials and performed the same regression analysis as described above for the original data. The cluster sums were determined, and the maximum absolute value cluster sum was stored for that shuffle. The process was then repeated while storing the maximum absolute value cluster sum across 1000 shuffles. These 1000 points made up the null test-statistic distribution that was expected when the relationship between LFP power and RT is random. All clusters in the original data with absolute values of sums falling above the 97.5 th or below the 2.5 th percentile of this empirical distribution were deemed to be significant.

For multiunit activity, to test its correlation with the arm movement reaction time, the reaction time was regressed onto three properties of the spike density functions (onset time, peak time, and slope):

$$
\mathrm{RT}=b_{0}+b_{1} \times X,
$$

where $X$ could be (1) the onset time of increases in firing rate: the time when the multiunit average firing rate passed two times the SD of the baseline firing rate; (2) the time of peak firing rate: the time when the multiunit activity reached its maximum firing rate; or (3) the resulting slope of the rise in firing rate: (maximum firing rate - onset firing rate)/(peak time - onset time). This was done separately for activity aligned to target onset and movement onset.
Sequential effects on LFP power. To investigate whether trial history had an influence on LFP power, we compared the LFP activity for go trials after canceled trials (Ca-Go) with go trials after go trials (Go-Go), and go trials after error trials (E-Go) with Go-Go trials. For this comparison, we first generated for each recording site groups of time-frequency arrays for $\mathrm{Ca}-\mathrm{Go}$, E-Go, and Go-Go trials aligned on target or movement onset, respectively. For this analysis, we pooled the two movement directions together to increase the available trial number. Next, we computed the mean difference between either Ca-Go and Go-Go, or E-Go and Go-Go arrays.

Then, we tested whether the differences in power for all pixels are significant by performing a permutation test separately for each pixel in the array (Efron and Tibshirani, 1993). During the test, we first randomly shuffled the LFP power values for that particular pixel across the two groups. In this way, we generated two groups that had the same size as the two original samples, but which contained values from trials with two different histories. Next, we calculated the difference of means between the two reshuffled groups. By repeating the resample process 10,000 times, we generated a permutation distribution under the null hypothesis $H_{0}$ that there is no effect of trial histories. By locating the sample mean on the permutation distribution, we estimated how rarely the difference of the sample means would occur under $H_{0}$ (i.e., the $p$ value for the pixel). By repeating the permutation test for all pixels of the arrays, we derived a map of $p$ values for the whole array.

The overall significance was corrected for multiple comparisons using the false-discovery rate (FDR) method (Benjamini and Yekutieli, 2001; Durka et al., 2004). First, the $p$ values of each pixel were ordered from smallest to largest: $p(1) \leq p(2) \leq \ldots p(k) \leq \ldots p(m)$. Next, we found the pixel with the largest rank order $k$, for which:

$$
p(k) \leq(k \times q) / m,
$$

where $q$ is the FDR-corrected $\alpha$ value $(q<0.05)$, and $m$ is the total number of comparisons on individual pixels. We assume here that the joint probability distribution of the pixels is non-independent. Then, the null hypothesis was rejected for all pixels with $p \leq p(k)$. We computed the grand average by computing the mean value of significant differences between different kinds of trials across all recording sites for each region and monkey.

Role of LFP activity in response inhibition. The countermanding task allowed us to determine whether the LFP contains signals sufficient to control the production of movement (Hanes et al., 1998; Scangos and Stuphorn, 2010). We compared canceled trials with latency-matched no-stop-signal trials, that is, the no-stop-signal trials with reaction time greater than the SSD plus the SSRT (Hanes et al., 1998). First, timefrequency arrays were generated for canceled and latency-matched nostop-signal trials separately for each movement direction and SSD. Next, we computed the difference between the two arrays, and aligned all difference arrays for one movement direction at the stop-signal onset. Then, we tested the significance of the difference in power for all pixels during the time period between SSD + SSRT $-70 \mathrm{~ms}$ and SSD + SSRT + 150 $\mathrm{ms}$ by performing a permutation test (10,000 repetitions), similar to the one used to test for history effects. We corrected for multiple comparisons using the FDR. After that, we computed the mean average of significant differences across all SSD for each recording site. The grand average showed that the mean significant differences in power align on SSRT across all recording sites. If the onset of significant differential activity occurred before the SSRT, we considered the LFP activity to contain signals that could causally contribute to the control of movement initiation.

\section{Results}

\section{Trial history influences behavior}

Both humans and monkeys show adjustments of saccade response time according to trial history (Emeric et al., 2007). In particular, response times increase after successive stop-signal trials and decrease after successive no-stop-signal trials. More generally, studies have shown response slowing following an error in choice tasks (Rabbitt, 1966). This observation has been 
A

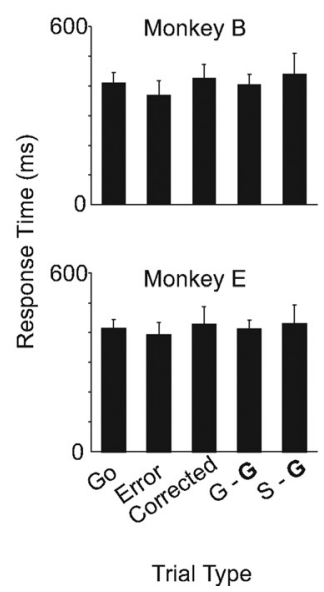

B

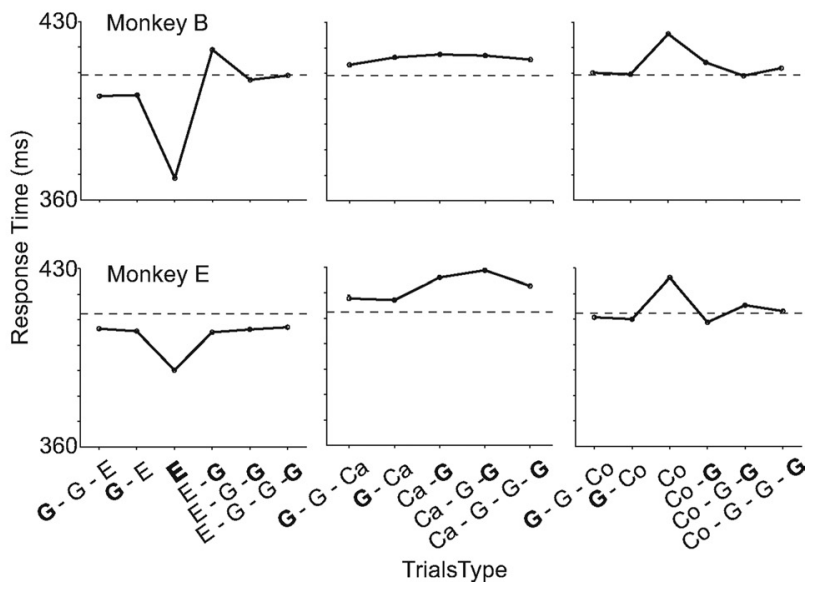

Figure 3. Effects of trial history on response time. $\boldsymbol{A}$, The mean response times on no-stop-signal trials, noncanceled trials, corrected trials, no-stop-signal trials that followed other no-stop-signal trials, and no-stop-signal trials that followed stop trials are shown for monkey B (top) and monkey E (bottom). Error bar represents the half SD. B, Response times for no-stop-signal and stop trials surrounding noncanceled trials (left), trials surrounding canceled trials (middle), and trials surrounding corrected trials (right) for monkey $B$ (top) and monkey $E$ (bottom). The type of trials to which the response time corresponds to is shown in bold (G: no stop signal; E: noncanceled; Ca: canceled; Co: corrected). The dotted line indicates the average response time on no-stop-signal trials.

stop trials (Fig. 3B, top middle and top right plots). The response time of nostop-signal trials following canceled trials was not significantly different from the response time of the preceding no-stopsignal trials (Go-Ca: 416 ms, Ca-Go: 417 $\mathrm{ms}, t$ test, $p=0.74)$. Since the monkey obtains reward on canceled trials, it appears that he adopts a strategy of maintaining his current average to slow response time after these trials.

Monkey E assumed a slightly different strategy (Fig. 3B, bottom row). As in monkey $\mathrm{B}$, the mean response time on noncanceled trials was significantly faster than on the preceding no-stop-signal trials (Go-E: $406 \mathrm{~ms}$, E: $390 \mathrm{~ms}$; $t$ test, $p<$ $0.0001)$. This trend of increasing speed was reversed on the next no-stop-signal trial that followed the noncanceled trial (E: $390 \mathrm{~ms}$, E-Go: $405 \mathrm{~ms}$; $t$ test, $p<$ 0.001). However, the posterror slowing effect was weaker than in monkey $\mathrm{B}$, since monkey E only returned to the speed level

regarded as evidence of executive control. We tested for similar behavioral adjustments in response to trial history in the arm stop-signal task.

On average, the movement latency on noncanceled trials was significantly faster than that on no-stop-signal trials, and movement latency on corrected trials was significantly slower for both monkeys (Fig. 3A) (monkey B: no-stop-signal trials 409 ms, noncanceled trials $368 \mathrm{~ms}$, corrected trials $464 \mathrm{~ms}$; monkey E: nostop-signal trials $415 \mathrm{~ms}$, noncanceled trials $390 \mathrm{~ms}$, corrected trials $483 \mathrm{~ms}$ ). We next examined how the no-stop-signal trial response time changed depending on the trial context. We found that in both monkeys the reaction time on no-stop-signal trials following other no-stop-signal trials (Go-Go) was faster than the reaction time on no-stop-signal trials following stop-signal trials (Stop-Go) (Fig. 3A) [monkey B: Go-Go: $414 \mathrm{~ms}$ (SD: $71 \mathrm{~ms}$ ), Stop-Go: 440 ms (SD: $142 \mathrm{~ms}$ ); monkey E: Go-Go: $410 \mathrm{~ms}$ (SD: $62 \mathrm{~ms}$ ), Stop-Go: $429 \mathrm{~ms}$ (SD: $128 \mathrm{~ms}$ )]. However, this trend did not reach significance (monkey B: $t$ test, $p=0.07$; monkey E: $p=$ $0.06)$. We then examined the reaction time on trials surrounding the different types of stop-signal trials.

For monkey B, we found that the average response time on no-stop-signal trials was $409 \mathrm{~ms}$ (Fig. 3B, top left plot, dotted line). The response times on no-stop-signal trials that preceded noncanceled by one or two trials were both slightly faster than average (Go-Go-E and Go-E: $401 \mathrm{~ms}$ ). Importantly, the average response time on noncanceled trials was much faster than the one on the preceding no-stop-signal trial (Go-E: $401 \mathrm{~ms}$, E: $368 \mathrm{~ms} ; t$ test, $p<0.0001$ ). Thus, the behavioral pattern shortly before and up to an error shows a pattern of increasing response speed. In contrast, the response time on the following no-stop-signal trials was significantly slowed (E-Go: $419 \mathrm{~ms}$ ), both relative to the noncanceled trials (E: $368 \mathrm{~ms} ; t$ test, $p<0.0001$ ) and relative to the last no-stop-signal trial before the error (Go-E: $401 \mathrm{~ms}$; $t$ test, $p<$ 0.0001 ). Thus, the monkey slowed down after an error, presumably in an attempt to improve subsequent performance (Fig. 3B, top left plot). Afterward, the monkey returned to a time closer to the average response time. These observations were similar to the posterror slowing found in other studies (Rabbitt, 1966; Emeric et al., 2007). This slowing was not observed after other types of of the no-signal trials before the error (Go-E: 406 ms, E-Go: 405 $\mathrm{ms} ; t$ test, $p=0.37$ ). On the other hand, monkey E responded to a canceled trial by reducing her response speed significantly relative to the no-stop-signal trial before the canceled trial (Go-Ca: $417 \mathrm{~ms}$, Ca-Go: $426 \mathrm{~ms}$; $t$ test, $p<0.001)$.

In summary, in both monkeys the response speed before a stop trial had a great impact on performance. Both monkeys responded faster than average before noncanceled trials, and slower than average before canceled trials. Furthermore, both monkeys adjusted their behavior in response to the outcome of the stop trial. Monkey B primarily reversed his response strategy following errors, while monkey E strengthened her response strategy following successes. Both strategies represent forms of proactive control, where the level of motor readiness is adjusted depending on past trial history.

\section{Movement-related activity pattern in LFP}

We first determined the general changes in LFP power across different frequency bands that accompanied the presentation of the target and the subsequent arm movement. Figure 4 shows the time-frequency maps of relative shifts in LFP power relative to baseline for two representative recording sites together with the simultaneously recorded multiunit activity.

The first recording was performed in the SMA of monkey B (Fig. 4A). The LFP activity showed two modulations. Starting just before target onset, there was a relative decrease in power in the low frequency range $(\sim 10-40 \mathrm{~Hz})$. This decrease was followed $\sim 100-120 \mathrm{~ms}$ later by a power increase in the high frequency range $(\sim 60-150 \mathrm{~Hz})$. The multiunit activity at this site was much stronger for contralateral movements and peaked before movement onset. This directional response was also visible in the LFP power in the high frequency range, however not in the low frequency range. Overall, the multiunit activity and the highfrequency LFP power seemed to be strongly positively correlated, and changes occurred simultaneously in both signals, while the low-frequency LFP power was more disassociated from the multiunit activity.

The second recording was performed in the SMA of monkey $\mathrm{E}$ (Fig. $4 B$ ). The main patterns of the temporal dynamic of the LFP 
A

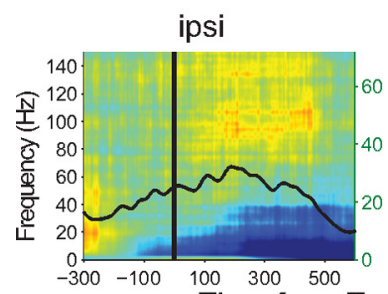

Time from Target Onset (ms)

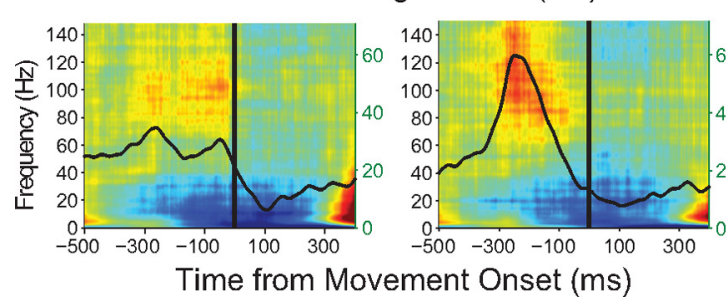

$\mathrm{B}$
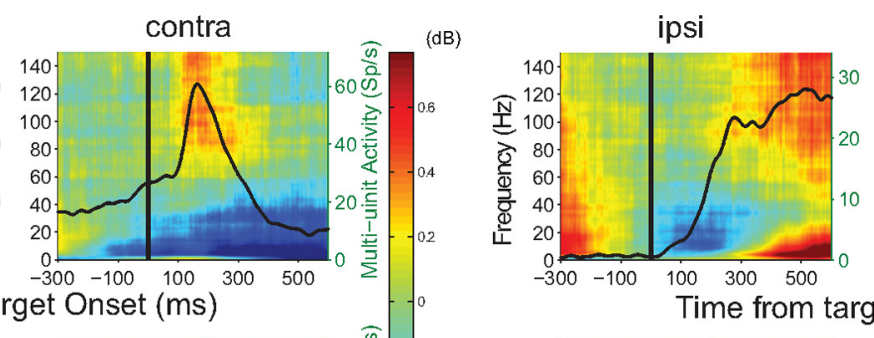

Monkey E
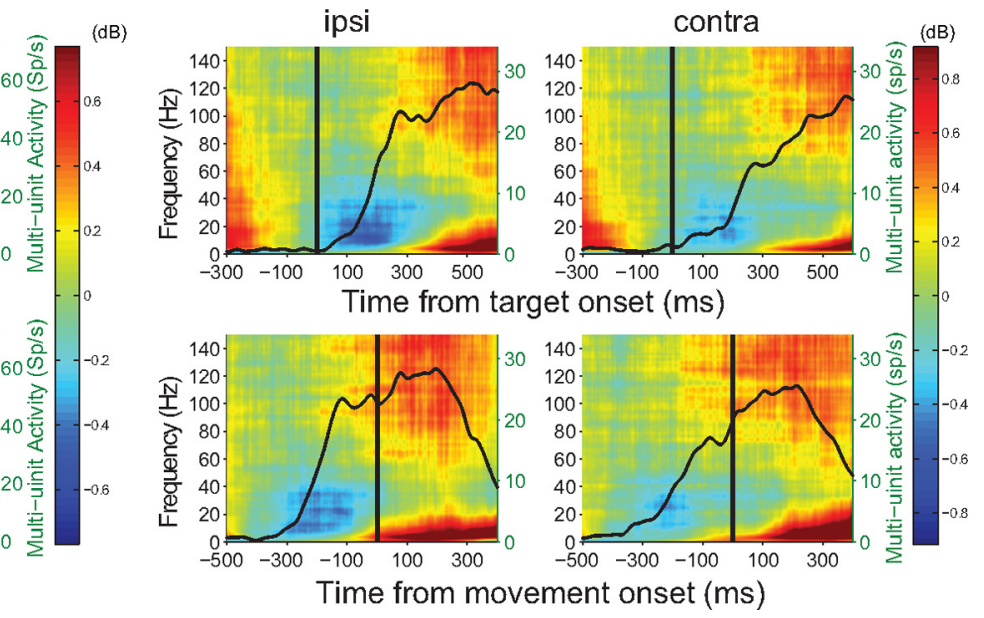

Figure 4. Examples of local field potentials and multiunit activity recordings. Time-frequency map of changes in the LFP power relative to baseline (in decibels), together with the firing rate of the simultaneously recorded multiunit activity (overlaid black curve). $\boldsymbol{A}$, Recording from the SMA of monkey B. $\boldsymbol{B}$, Recording from the SMA of monkey E.

A

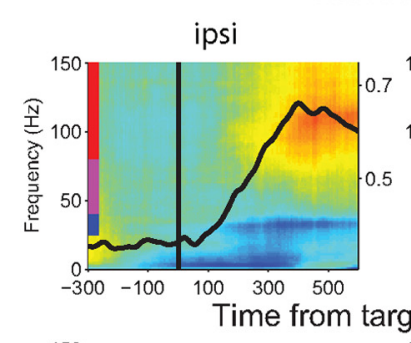

Monkey B
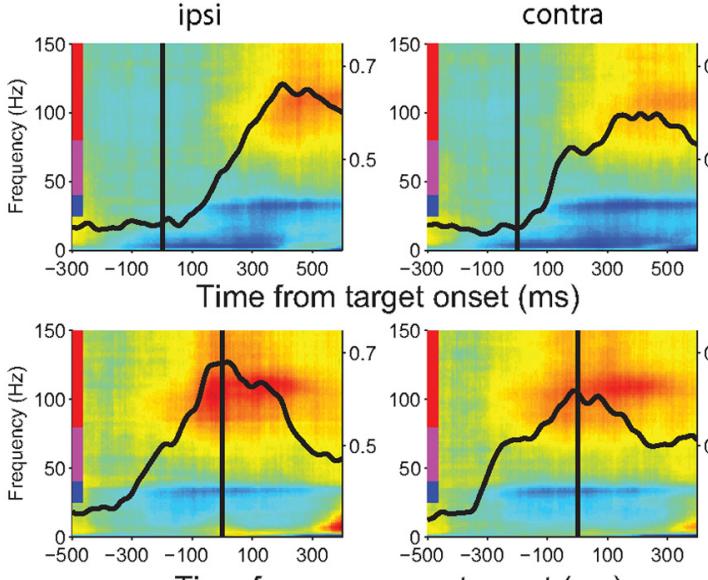

Time from movement onset $(\mathrm{ms})$
B

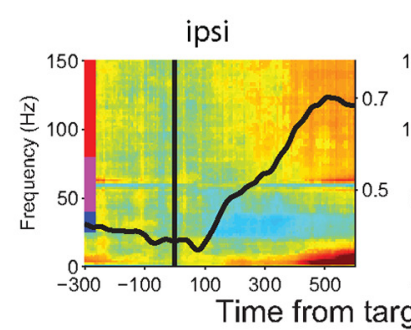

Monkey E
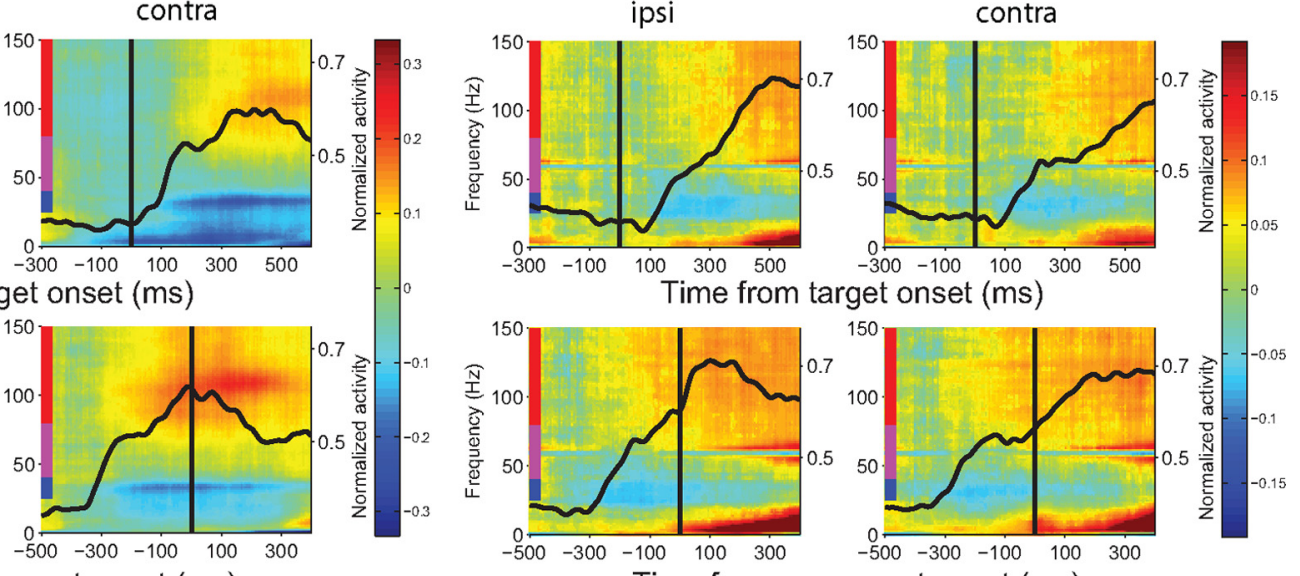

Time from movement onset (ms)

Figure 5. Grand average of local field potentials and multiunit activity. The resulting time-frequency maps of the normalized change in LFP power and multiunit activity (overlaid black curve) across all the recordings for ipsilateral movements (left column) and contralateral movements (right column) are shown aligned on both target presentation (up) and movement initiation (bottom). $\boldsymbol{A}$, Grand average of all recordings from SMA of monkey B. $\boldsymbol{B}$, Grand average of all recordings from SMA of monkey $E$.

power and its correlation with multiunit activity were the same. Immediately after target onset, the low-frequency LFP power decreased, followed after $\sim 200 \mathrm{~ms}$ by an increase in high-frequency LFP power. Also, the multiunit activity was best correlated with the high-frequency LFP power. However, there were also some differences. Most notably, there was increased LFP power in a very low-frequency range $(\sim 1-20 \mathrm{~Hz})$ around the same time that there was increased power in the high-frequency range, which was completely absent in the recording from monkey B. Furthermore, the relative decrease in low-frequency $(10-40 \mathrm{~Hz}) \mathrm{LFP}$ power was shorter in the second recording and lasted only during the time period between target onset and movement onset.

We normalized and averaged LFP power and multiunit activity to determine which activity patterns were consistent across recordings. Figure 5 shows the resulting time-frequency maps of the average change in LFP power and multiunit activity for SMA recordings from monkey $\mathrm{B}$ and $\mathrm{E}$. The findings confirmed many of the findings in the individual recordings. We observed in both monkeys a reduction of low-frequency $(10-40 \mathrm{~Hz})$ LFP power and an increase in high-frequency $(60-150 \mathrm{~Hz})$ LFP power (Fig. 5A: monkey B; Fig. 5B: monkey E).

In particular, the temporal dynamic of the changes in highfrequency power was very similar in both monkeys. The SMA is part of the motor system and neuronal activity is mostly directional (Scangos and Stuphorn, 2010). We therefore analyzed the LFP power separately for arm movements in both directions. Overall, the temporal dynamics of the LFP power for ipsilateral and contralateral arm movements were similar, but ipsilateral movements elicited slightly larger increases in power. The power in the high-frequency band continued to increase and reached a maximum in the time around movement onset (Fig. 5A) (monkey B) or shortly after (Fig. 5B) (monkey E).

In contrast, the temporal dynamic of the LFP across the lowfrequency band showed some differences between the two monkeys. In both monkeys, there was a decrease in power in the frequency band between 25 and $40 \mathrm{~Hz}$. The onset of the power reduction in this frequency band was 100-50 ms before target onset and clearly preceded the increase in high-frequency power. 
Table 1. Spearman rank correlation coefficients for correlation between multiunit activity and LFP power in three different frequency bands

\begin{tabular}{|c|c|c|c|c|c|c|}
\hline & \multicolumn{2}{|c|}{ High gamma $(80-150 \mathrm{~Hz})$} & \multicolumn{2}{|c|}{ Gamma $(40-80 \mathrm{~Hz})$} & \multicolumn{2}{|c|}{ High beta $(25-40 \mathrm{~Hz})$} \\
\hline & Target & Move & Target & Move & Target & Move \\
\hline Monkey B SMA left & 0.9614 & 0.9623 & 0.8519 & 0.8776 & -0.9707 & -0.9624 \\
\hline Monkey B SMA right & 0.9537 & 0.9623 & 0.8345 & 0.9175 & -0.9156 & -0.9571 \\
\hline Monkey E SMA left & 0.9475 & 0.9312 & 0.8242 & 0.6602 & -0.3491 & -0.7056 \\
\hline Monkey E SMA right & 0.9389 & 0.8651 & 0.8328 & 0.7077 & 0.2565 & -0.6449 \\
\hline
\end{tabular}

The duration of this reduction in low-frequency LFP power was much longer in monkey $\mathrm{B}$, where it lasted for as long as the duration of the increase in high-frequency LFP power. In monkey $\mathrm{E}$, the decrease ceased shortly after the onset of the arm movement.

In summary, the LFP power in the SMA showed a consistent pattern of dynamic changes across both monkeys. First, LFP power in a low $(25-40 \mathrm{~Hz})$-frequency band decreased, starting immediately before or at the moment of target onset. This decrease was followed by an increase of LFP power in a high (60$150 \mathrm{~Hz}$ )-frequency band. These observations are in accord with existing findings concerning the pattern of electrocorticographic activity in the motor cortex of humans during simple movements (Pfurtscheller et al., 1994, 2003; Crone et al., 1998a,b). In the following analysis, we will concentrate on these two high- and low-frequency bands.

\section{Correlation between multiunit activity and LFP power}

Across all recordings, the temporal dynamic of the multiunit activity was most similar to the dynamic of the high-frequency LFP power. To quantify the relationship between multiunit spiking activity and different components of the LFP, we computed the cross-correlation between mean normalized firing rates and mean normalized LFP power in the high- and low-frequency band aligned on target and movement onset for ipsilateral and contralateral movements (supplemental Fig. 1, available at www. jneurosci.org as supplemental material). In general, the crosscorrelation analysis confirmed that the high-frequency LFP power was positively correlated, and the low-frequency LFP was negatively correlated with multiunit activity. As shown in Table 1, the correlation strength was higher for high- as compared to low-frequency LFP modulation, especially for monkey E. Because of the broad peaks in both multiunit activity and LFP modulation, there was rarely a clear latency at which the crosscorrelations reached a peak. This was particular true when both signals were aligned on movement onset. The correlations between high-frequency LFP power and multiunit activity tended to be largest for negative to no lag. This indicated that changes in firing rate preceded or occurred synchronously with similar changes in high-frequency LFP power. The correlations between low-frequency LFP power and multiunit activity tended to be largest for positive to no lag. This indicated that decreases in low-frequency power were followed by increases in multiunit activity.

Thus, the main findings of our study are in agreement with findings in earlier studies that looked at the relationship between spiking activity and different frequency components of the LFP (Ray et al., 2008b), and extends it to frontal, agranular cortex recordings. Specifically, our findings support the hypothesis that LFP high-frequency $(60-150 \mathrm{~Hz})$ activity is a measure of the number of action potentials generated by the neuronal population near the electrode tip (Liu and Newsome, 2006; Ray et al., 2008a,b). However, the LFP activity most likely reflects activity in a larger cortical volume than the multiunit activity (Berens et al.,
2008). In the remaining text, we will refer to the $60-150 \mathrm{~Hz}$ frequency range as "high gamma frequency." In contrast, the LFP low-frequency $(25-40 \mathrm{~Hz})$ activity most likely reflects synaptic activity (Mitzdorf, 1985; Viswanathan and Freeman, 2007). We will refer to this frequency range as "beta frequency." For a more detailed discussion of the functional interpretation of LFP power in different frequency bands, see the supplemental material (available at www.jneurosci.org).

\section{Variation in LFP power is correlated with reaction time}

In the stop-signal task, there is an incentive for the monkeys to control the speed with which arm movements are generated in response to the perceived likelihood of the appearance of a stop signal. Indeed, our behavioral analysis showed that both monkeys adjust behavior according to the previous trial type to some extent. For the first monkey in particular, response times slowed after an erroneous noncanceled trial. These findings indicate that a proactive control system uses response history and trial outcome to adjust behavioral strategy over a series of trials.

Recent recording and microstimulation experiments suggest that the medial frontal cortex plays an important role in the executive control of motor behavior (Stuphorn and Schall, 2006; Isoda and Hikosaka, 2007; Stuphorn et al., 2010). We were therefore interested to examine whether changes in neural activity in SMA were correlated with these behavioral changes. We first tested whether changes in LFP power were related to the monkey's response speed in all 47 LFP recordings in the SMA aligned on target and movement onset. To test the effect on movement generation without the additional complication of an intervening inhibitory process, we concentrated this regression analysis on no-stop-signal trials.

We started by analyzing the correlation between the LFP power and arm movement reaction time aligned on target onset. This analysis showed that the LFP power in the beta and high gamma frequency range in the SMA was related to arm movement reaction time. For each recording, we computed a timefrequency map of the relative change in LFP power across a $0.7-150 \mathrm{~Hz}$ frequency band for the -300 to $600 \mathrm{~ms}$ time period around target onset. To visualize the shifts in LFP power across the time-frequency map that accompany changes in reaction time, we divided the no-stop-signal trials by reaction time into three equally sized groups and computed time-frequency LFP power maps separately for each group: fast, medium, and slow responses. Figure 6 shows the result of this analysis for all SMA recordings in monkey $\mathrm{B}$. Two trends are apparent. First, the onset, but not the dynamics, of the pattern of changes in LFP power during arm movement generation shifts with reaction time. The decrease of power in the beta frequency, followed by the increase in the high gamma band, occurs earlier in trials with fast responses (Fig. 6, left column), and later in trials with slow responses (Fig. 6, right column). Second, the relative amount of power in the high gamma range in the $200 \mathrm{~ms}$ before target onset is inversely correlated with reaction time. In other words, the 
A Monkey B
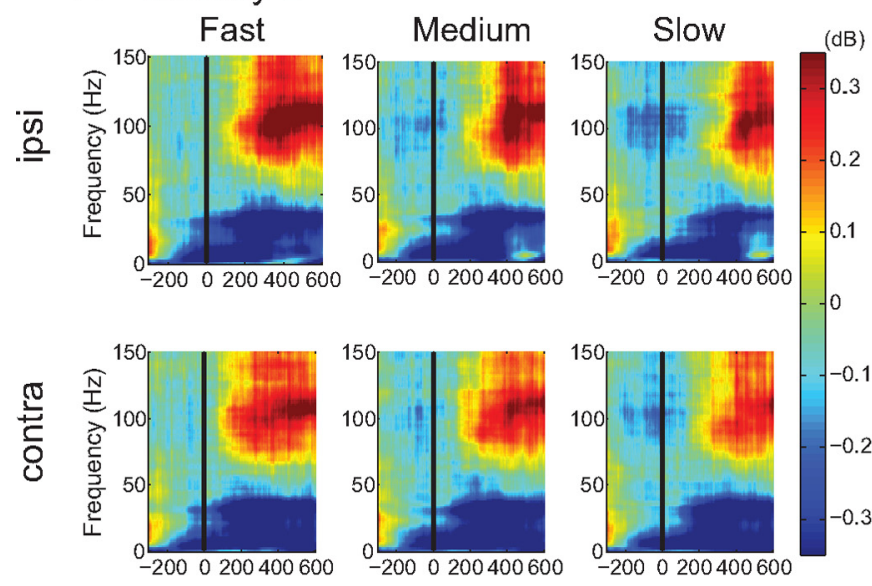

Time from target onset (ms)
B Monkey E Fast
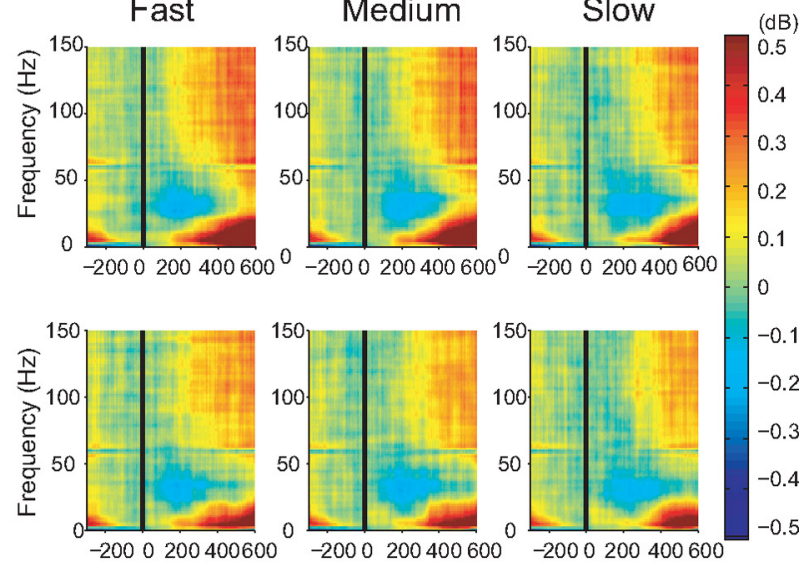

Time from target onset (ms)

Figure 6. Grand average of local field potential aligned on target presentation with fast, medium, and slow responses. The upper row shows ipsilateral movements, and the lower row shows contralateral movements across all recordings. $\boldsymbol{A}$, Grand average of all recordings from SMA of monkey B. B, Grand average of all recordings from SMA of monkey E.

stronger the depression in this time-frequency region, the later the monkey responded.

Next, we performed a linear regression between the LFP power and the arm movement reaction time for each individual pixel in the time-frequency map. The upper plots of Figure $7 \mathrm{~A}$ show the mean slope of the linear regression between the LFP power and the arm movement reaction time across all SMA recordings in monkey B. The results for ipsilateral and contralateral movements are shown separately. The lower plots of Figure $7 \mathrm{~A}$ shows the same time-frequency map of regression slopes when we only took significant slope values into account. There are a number of different time-frequency regions, where LFP power is consistently related to reaction time. In particular, activity in the beta band is positively correlated with arm movement reaction time, while activity in the high gamma band is negatively correlated with arm movement reaction time. Significant regressions in both frequency bands started 300-250 ms before target onset. At this point, the monkey did not yet know the direction of the next upcoming arm movement, and it is therefore impossible that this modulation reflect shifts in the timing of motor processes. This early relationship between LFP power and later reaction time is therefore strong evidence that the LFP power modulation reflects a proactive control signal in preparation of the upcoming task. In addition to the early correlation, there is also an later area of negative correlation in the beta band between 7 and $20 \mathrm{~Hz}$ that started after target onset and which lasted for 400 ms. This pattern is present both for ipsilateral and contralateral movements, but the correlation strength is slightly stronger for ipsilateral movements. The LFP power recorded in the SMA of monkey E showed similar effects (Fig. $7 B$ ). Activity in the highgamma band was negatively and activity in the beta band was positively correlated with reaction time. In both cases, the significant correlation started before target onset, in particular the activity in the beta band.

So far, the regression analyses were performed on activity aligned on target onset. We then asked whether LFP activity was also correlated with arm movement reaction time when we aligned it on movement onset. Figure 8 shows the time-frequency LFP power maps for three equal-sized response time groups: trials with fast (left), middle (medium), and slow (right) responses for the SMA in monkey B. The pattern of activity in all three groups was very similar. In particular, the onset of the activity pattern in the LFP was time locked with arm movement initiation, regardless of the reaction time. The decrease in the beta band started $400 \mathrm{~ms}$ before movement onset, while the increase in the high-gamma band started $300 \mathrm{~ms}$ before movement onset. However, there was a weak positive relationship between the intensity of the decrease and increase in LFP power and reaction time, in particular for ipsilateral movements.

We performed a linear regression between LFP power and both movement onset and reaction time for each pixel of the time-frequency map. The test of regression covered a $0.7-150$ $\mathrm{Hz}$ frequency range in a time range of $-500 \mathrm{~ms}$ to $400 \mathrm{~ms}$ relative to movement onset. For both monkeys $\mathrm{B}$ and $\mathrm{E}$, the LFP power in a large number of pixels was significantly correlated with movement initiation time (Fig. 9). However, there were a number of differences from the result when the same trials were aligned on target onset. First, the regression between LFP power and reaction time was less strong. Second, LFP power across a wide range of times and frequencies formed a more irregular pattern of pixel with significant correlations without clear clusters. Third, the regression between high gamma LFP power and reaction time was often positive. Thus, while an early onset of the activity burst in the high gamma range was correlated with a fast reaction time (Fig. 6), once the activity burst had started, more high gamma LFP power was correlated with a slower reaction time (Fig. 8).

Thus, our findings indicate that LFP power in the beta and high gamma range in the SMA is correlated with arm movement reaction time even before target onset. Less power in the high gamma range and more power in the beta range lead to longer reaction times. The fact that such early LFP modulations seem to influence arm movement generation is an indication that SMA proactively controls the state of excitability in the skeletomotor system. The pattern of LFP power during the actual arm movement preparation and execution was also related to reaction time. This seems to indicate that the proactive control of excitability continued throughout the entire movement generation. However, overall the onset of the pattern in LFP power had a much greater influence on reaction time than its dynamics. 

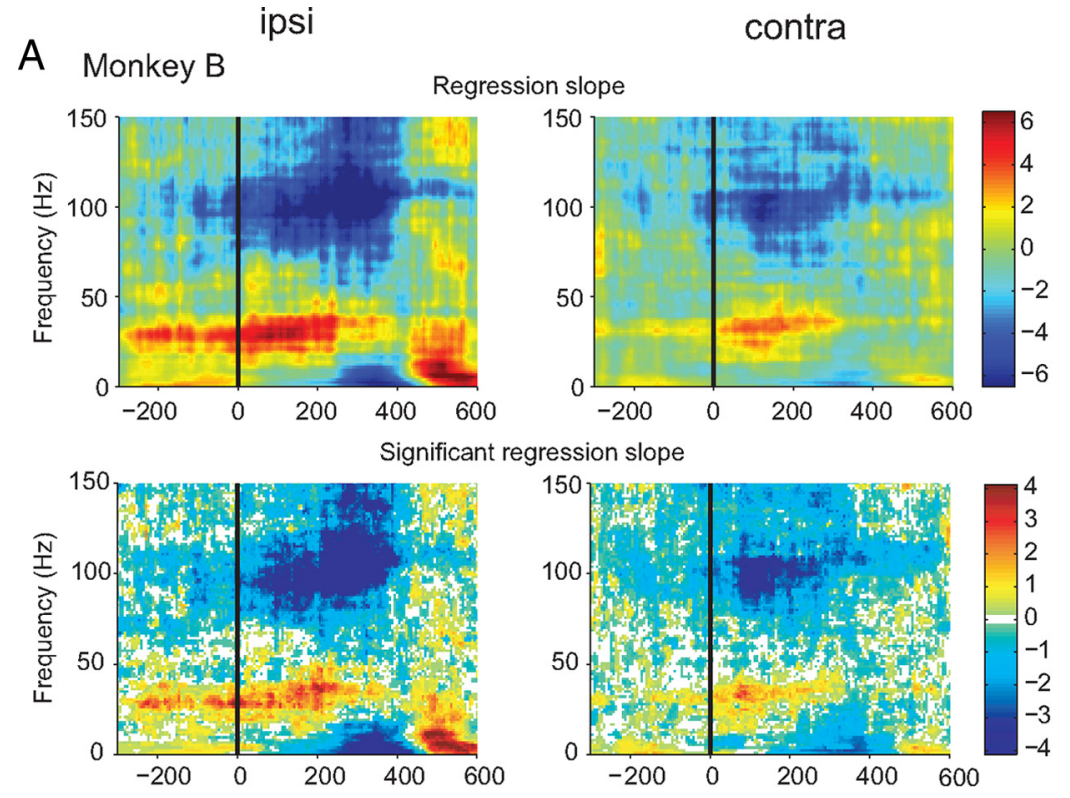

B Monkey E
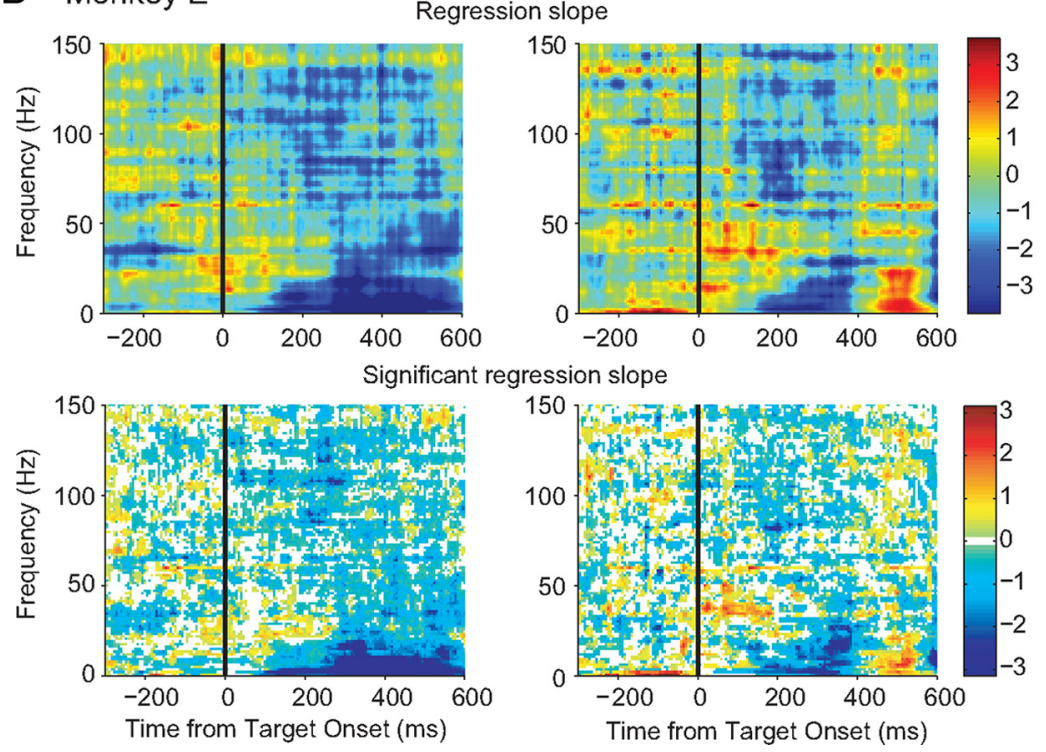

Figure 7. Time-frequency map of the mean regression slope between reaction time and LFP power recorded in SMA aligned on target onset. The upper panels plot the mean slope of the linear regression between the LFP power and arm movement reaction time across all recordings from SMA of monkey B. The lower panels show the mean slope of only the significant regressions. The left panels plot ipsilateral movements, while the right panels plot contralateral movements. A, Monkey B. B, Monkey E.

Onset of multiunit activity is correlated with reaction time

Since there is a close correlation between LFP power in the high gamma band and multiunit activity, we tested whether the dynamics of SMA multiunit activity was related to reaction time, as well. For each recording, we sorted the no-stop-signal trial by reaction time into five different groups with equal number of trials (from fastest to slowest) and determined whether some properties of the average spike density function across the five groups correlated with reaction time. Specifically, we determined the onset of increases in firing rate, the time of peak firing rate, and the resulting slope of the rise in firing rate separately for ipsilateral and contralateral movements and aligned the activity both on target onset and on movement onset.

Figure $10 \mathrm{~A}$ shows a representative example of multiunit activity in SMA aligned on target onset for ipsilateral movements, which was the preferred direction for this site. Across the five groups of trials, the onset and peak of movement-related activity systematically varied with increasing reaction time. In contrast, the slope of the rise in multiunit activity was very similar for four of the five groups. Only the slope of the trials with the earliest onsets was less steep than the slopes of the other four groups of trials. Figure $10 C$ shows the result of a regression between reaction time and activity onset, peak, and slope. Both the timing of the onset and the peak of the movement relative activity were significantly correlated with the reaction time of arm movement (onset: $p<0.001$; peak: $p<0.01$ ). Slope, however, was not significantly correlated with reaction time $(p=$ 0.052 ) and would have been nearly constant without the outlier from the group with the fastest arm movements. Thus, the onset of multiunit activity seemed to determine primarily the speed with which an arm movement is generated. Following the onset, the multiunit activity increased with a constant rate and peaked at the same time relative to movement onset. This observation was confirmed when the multiunit activity was aligned on movement onset (Fig. $10 \mathrm{~B}$ ). The pattern of activity relative to movement onset in all five groups was very similar. Neither the timing of the onset and the peak of this activity nor the slope of its rise was significantly correlated with reaction time (onset: $p=$ 0.14; peak: $p=0.09$; slope: $p=0.18$ ) (Fig. $10 D)$. Thus, at this recording site, the onset but not the slope of the rise in multiunit activity seemed to be related to movement onset.

The results from the multiunit recordings across the 47 SMA recordings agreed with this hypothesis concerning the relationship between population activity and arm movement reaction time (Fig. 11). The results for the multiunit activity recorded in the SMA of the two monkeys were not significantly different and were combined. The onset of multiunit activity relative to target onset tended to be positively correlated with reaction time (mean coefficient: $0.293 ; t$ test, $p<0.04$ ). The slope of the rise in activity, on the other hand, tended to be constant (mean coefficient: $6.4 \times 10^{-5} ; t$ test, $p=0.53$ ), so that the time at which the activity peaks relative to target onset is also significantly correlated with reaction time (mean coefficient: $0.424 ; t$ test, $p<0.01$ ). Neither onset and the timing of peak activity nor the slope of rise in activity tended to be correlated with reaction time, when activity was aligned on movement onset (Table 2). Altogether, these results suggest that the rise of multiunit activity in the SMA follows a stereotypical firing pattern before arm movement initiation. Reaction time was related to the onset of the rise in multiunit activity, but not its slope. 


\section{A Monkey B}
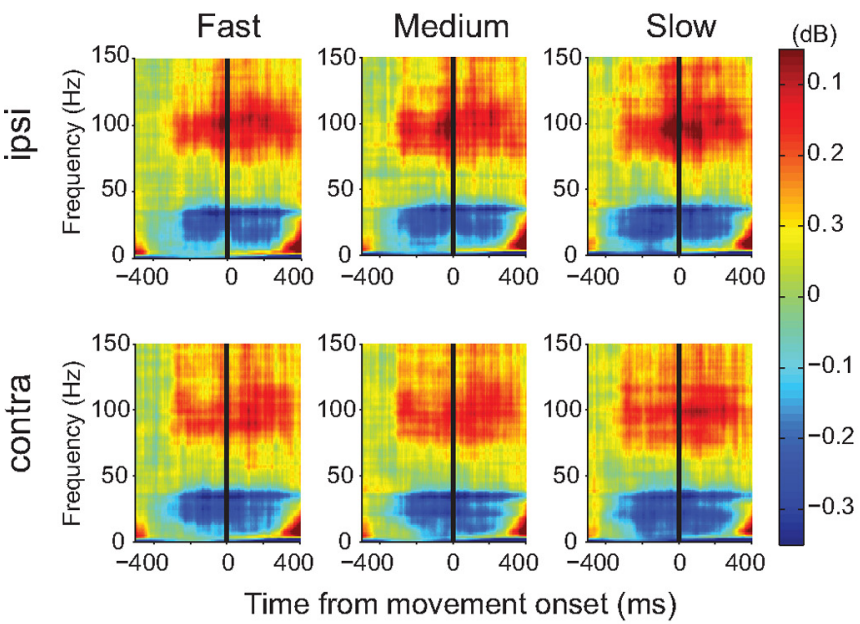
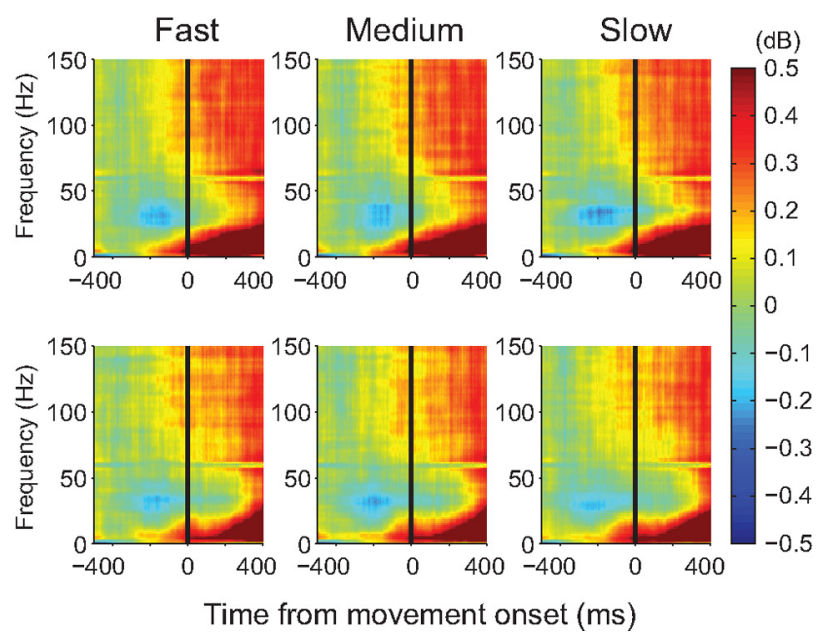

Figure 8. Grand average of local field potentials of monkey B aligned on movement initiation with fast, medium, and slow responses across all SMA recordings. Conventions are as in Figure 6 . $A$, Monkey B. B, Monkey E.

\section{Trial history influences LFP activity}

LFP and multiunit activity in the SMA are correlated with arm movement response time, which is a function of the level of excitability in the primary motor cortex (M1). A high level of motor excitability leads to faster and stronger EMG activation, which in turn, leads to faster movement generation (shorter reaction time). We hypothesize that the population activity of neurons in the SMA, as indexed by LFP and multiunit recordings, may proactively regulate the M1 excitability level. By setting the balance of excitation and inhibition, SMA neurons could regulate how fast motor neurons in M1 respond, and thus influence reaction time.

To test this possibility, we first determined whether trial history had an influence on LFP activity. Behaviorally, the monkeys changed their reaction time following a stop-signal trial (Fig. 3). If the SMA activity reflects proactive control, we would expect to see changes in LFP power in a way that would explain the effects of trial history on behavior. Specifically, we compared the LFP activity on no-stop-signal trials that followed another no-stopsignal trial (Go-Go) with LFP activity on no-stop-signal trials that followed either a canceled trial $(\mathrm{Ca}-\mathrm{Go})$ or a noncanceled trial (E-Go). For each recording, we compared each individual pixel of the two time-frequency maps of LFP power aligned on target onset using a permutation test. We adjusted for multiple comparisons using the FDR procedure. Since the behavioral trial history effects did not depend on the direction of the consecutive arm movements, in this analysis we combined trials with ipsilateral and contralateral movements. The analysis showed that the LFP power particularly in the low beta frequency range was modulated by trial history.

Figure 12 shows the time-frequency maps of the mean LFP power during Go-Go, Ca-Go, and E-Go trials, and the significant differences between them across all SMA recordings in monkey $B$. First, we compared the time-frequency maps of all trial types aligned on target onset (Fig. 12A,B). Canceled trials led to increased LFP power in the beta band $(25-40 \mathrm{~Hz})$ in the following no-stop-signal trials (Ca-Go trials) (Fig. 12A). In addition, there was a slight increase of activity in a very low-frequency band (1-20 Hz), which started $\sim 200 \mathrm{~ms}$ before target onset. In comparison, noncanceled trials had a much larger influence on the LFP power activity in the following no-stop-signal trials (E-Go trials) (Fig. 12B). In particular, there was a strong increase of activity in the very low-frequency band $(1-20 \mathrm{~Hz})$ and a less pronounced increase in the beta band $(25-40 \mathrm{~Hz})$ starting $\sim 120$ $\mathrm{ms}$ before target onset. This stronger effect on E-Go trials fits the behavioral effects seen in monkey B, which showed a large increase in reaction time following an error, but a more modest adjustment of reaction time following a successfully canceled stop-signal trial (Fig. 3).

The increase of low-frequency LFP power in E-Go trials relative to Go-Go trials is consistent with our previous results. The onset of change in LFP power in SMA shifted with reaction time (Fig. 6). Since monkey B markedly slowed down after errors (Fig. $3 B$ ), the increase of activity in the low-frequency band likely reflects in part the later onset of the premovement activity on those trials. However, this raises the question of whether the history effects on LFP power indeed represent the proactive control signals themselves, or whether they are merely a reflection of the shifts in reaction time.

We therefore next aligned the LFP power time-frequency maps on movement onset, to account for any shifts in reaction time, before taking the difference between the power maps to isolate history effects (Fig. 12C,D). All effects were confirmed, and in fact became more noticeable, when we aligned the timefrequency maps on movement onset. In Ca-Go trials, there was an increase of LFP power in the beta band $(25-40 \mathrm{~Hz})$ and in a low-frequency band $(1-15 \mathrm{~Hz})$. In E-Go trials, there was an increase in both of these frequency bands as well. This finding confirmed that history effects were present in LFP power that could not be explained by a shift in the timing of the motor response across conditions relative to the onset of the visual target.

Figure 13 shows the sequential analysis of the LFP power time-frequency maps across all SMA recordings in monkey E. In Ca-Go and E-Go trials, the LFP power recorded in the SMA showed an increase in the low-frequency band and especially in the beta band as early as $12 \mathrm{~ms}$ after target onset, when the timefrequency maps were aligned on target onset (Fig. 13A, B). This effect is similar to the one shown in monkey B. In addition, there was also a mixture of increases and decreases of LFP power in different sections of the high gamma frequency band. There was also a relative decrease in LFP power in the low-frequency band $(1-40 \mathrm{~Hz})$ in both Ca-Go and E-Go trials. This relative decrease 
A ipsi

A Monkey B

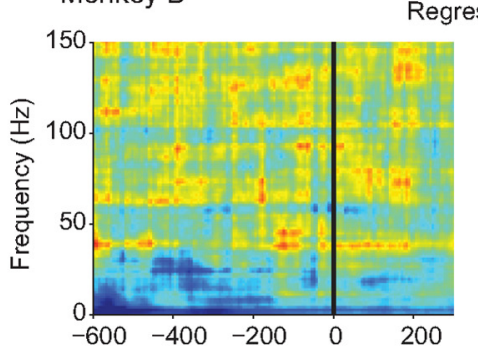

Regression slope

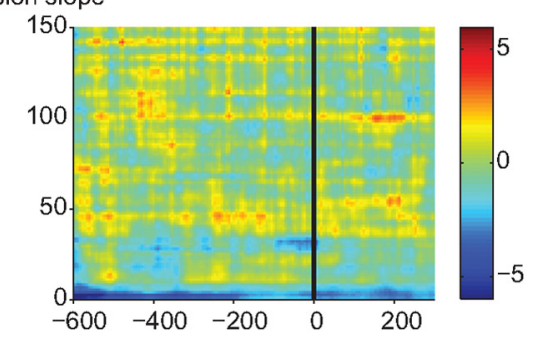

Significant regression slope
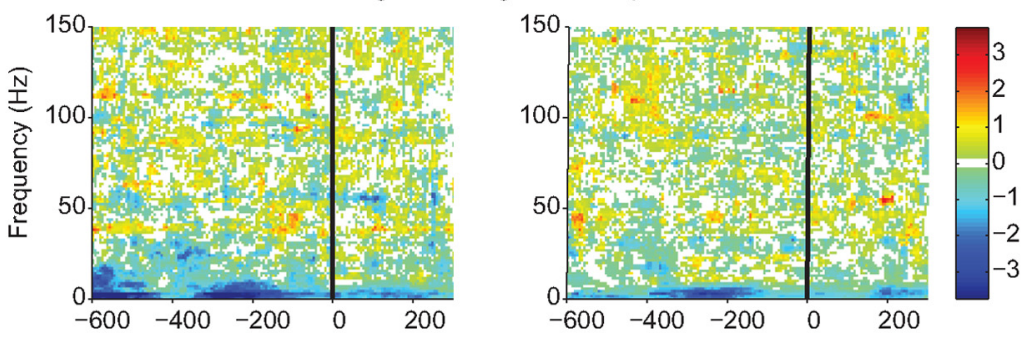

B Monkey $\mathrm{E}$

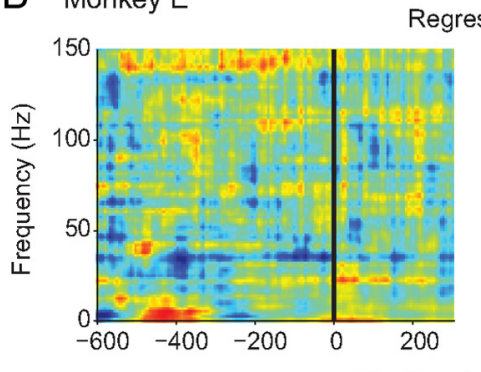

Regression slope

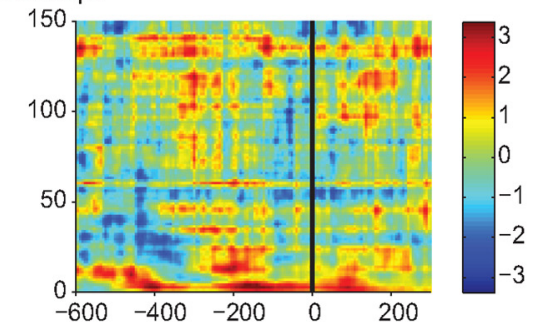

Significant regression slope
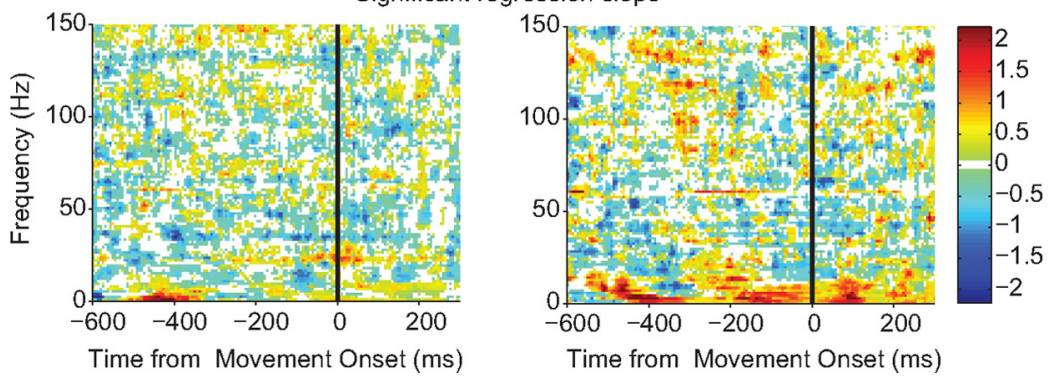

Figure 9. Time-frequency map of the mean regression slope between reaction time and LFP power recorded in SMA aligned on movement onset. Conventions are as in Figure 7. A, Monkey B. B, Monkey E.

in LFP power is the result of a shift in the onset of increase in LFP power, which is specific to monkey E (Figs. 4, 5). All effects found in the time-frequency maps of LFP power aligned on target onset were confirmed when we aligned the time-frequency maps on movement onset (Fig. 13C,D). The increases of activity in the beta band and especially the broad pattern of increases and decreases in the high-gamma band were much more pronounced in Ca-Go trials than in E-Go trials. This finding fits with the behavioral pattern in monkey $\mathrm{E}$, which showed an increase in reaction time following a successfully canceled stop-signal trial, but a more modest adjustment of reaction time following an error (Fig. 3).

In summary, the LFP recordings in the SMA did show effects of trial history. These modulations of LFP power likely represent ongoing differences in the state of excitability, resulting in the differences in reaction time. They therefore indicate that the proactive control signals in the SMA are long-lasting and tonic in nature. That distinguishes them from the phasic reactive control that is exerted in response to the sudden occurrence of a stop signal. Most of the trial history effects on LFP power involved very low-frequency bands, while the effects in the high gamma range were weaker and more diffuse, in particular in monkey B. High gamma activity seems to reflect mostly spiking activity of neurons in the vicinity of the electrode tip, while lower frequencies seem to reflect mostly synaptic input and dendritic currents. Thus, the LFP analysis showed that adjustments in proactive control in the SMA lead to large changes in synaptic input and in internal computation within local SMA neurons. Changes in firing rate, on the other hand were harder to detect in the LFP recordings.

\section{Trial history influences multiunit activity}

If SMA indeed proactively controls the level of excitation in the motor cortex, it needs to be able to influence motor cortex. This would require the generation of action potentials. Therefore, the changes in synaptic activity reflected in the LFP should also lead to change in the firing rate of neurons. We tested the influence of trial history on neuronal activity in SMA by analyzing multiunit activity. Similar to our LFP analysis, we compared the multiunit activity on Go-Go trials with the activity on Ca-Go trials and E-Go trials, respectively. We analyzed the mean firing rate in three different periods of each trial: a baseline time period (200 ms before target onset), a period after target presentation $(0-400 \mathrm{~ms}$ following target onset), and a period closely related to the arm movement ( -200 to $200 \mathrm{~ms}$ relative to movement onset). We quantified the change in firing rate by computing a sequential effect index for each time period: $I=\left(\mathrm{MU}_{\mathrm{Ca}-\mathrm{Go}}-\mathrm{MU}_{\mathrm{Go}-\mathrm{Go}}\right) /\left(\mathrm{MU}_{\mathrm{Ca}-\mathrm{Go}}+\right.$ $\mathrm{MU}_{\mathrm{Go}-\mathrm{Go}}$ ), where $\mathrm{MU}_{\mathrm{Ca}-\mathrm{Go}}$ indicates the mean multiunit activity in the trial period on Ca-Go trials and $\mathrm{MU}_{\mathrm{Go}-\mathrm{Go}}$ indicates the mean activity on Go-Go trials. The significance of the differences in mean discharge rate in the three trial periods were tested using a bootstrapping test.

The results showed that in a substantial number of cases, preSMA and SMA multiunit activity significantly changed its firing rate depending on the immediate trial history. These effects could involve either an increase or a decrease of activity and could be present either on Ca-Go, E-Go, or both trial types. Figure 14 shows two representative examples from the SMA of monkey B indicating the range of effects. The activity on Go-Go trials is shown in black, while the activity on Ca-Go trials (upper panels) and E-Go trials (lower panels) is shown in red. The baseline period is indicated by the black horizontal line in the panel on the left hand side, while the target onset period is indicated by the red horizontal line. The movement time period is indicated by the longer black line in the panel on the right hand side. During E-Go 
A

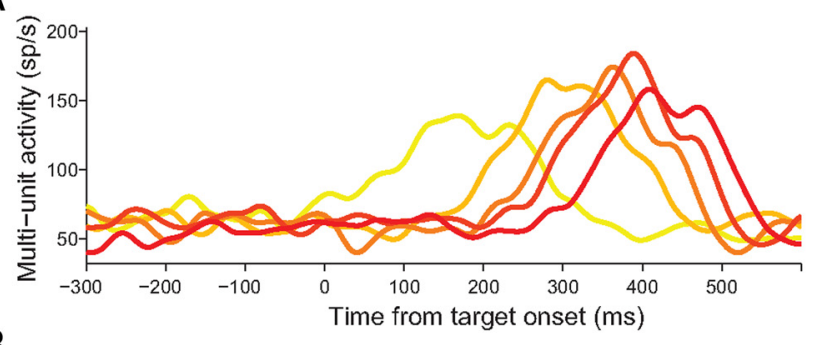

$\mathrm{B}$

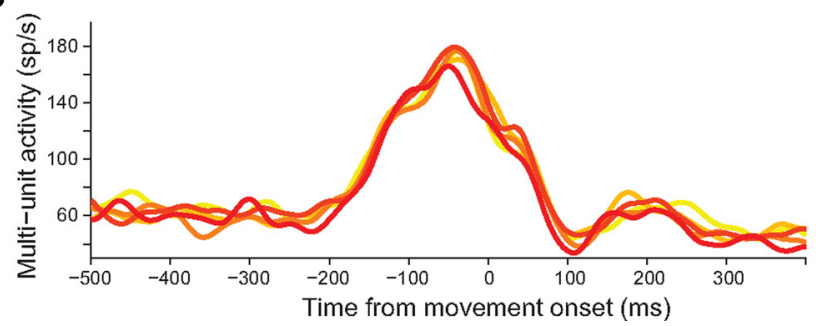

C
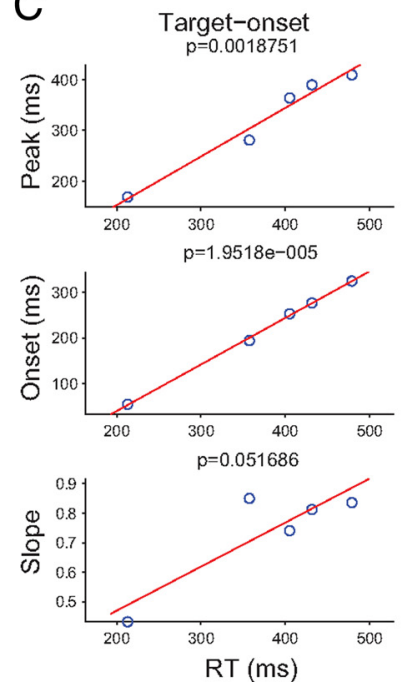
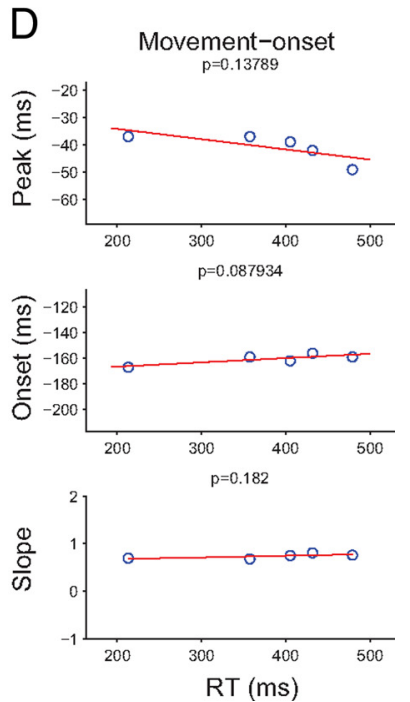

Figure 10. Relationship of multiunit activity to reaction time. The activity of a representative SMA multiunit activity is illustrated aligned on target onset $(\boldsymbol{A})$ and on arm movement initiation $(\boldsymbol{B})$ for its preferred direction. All trials with no stop signals were divided into five groups with equal number of trials according to arm movement response time from fastest (yellow) to slowest (red). C, The result of the regressions between the timing of the reaction time and the timing of the activity onset, the timing of the peak activity, and slope when aligned on target onset. $\boldsymbol{D}$, The same regression results when aligned on arm movement initiation.

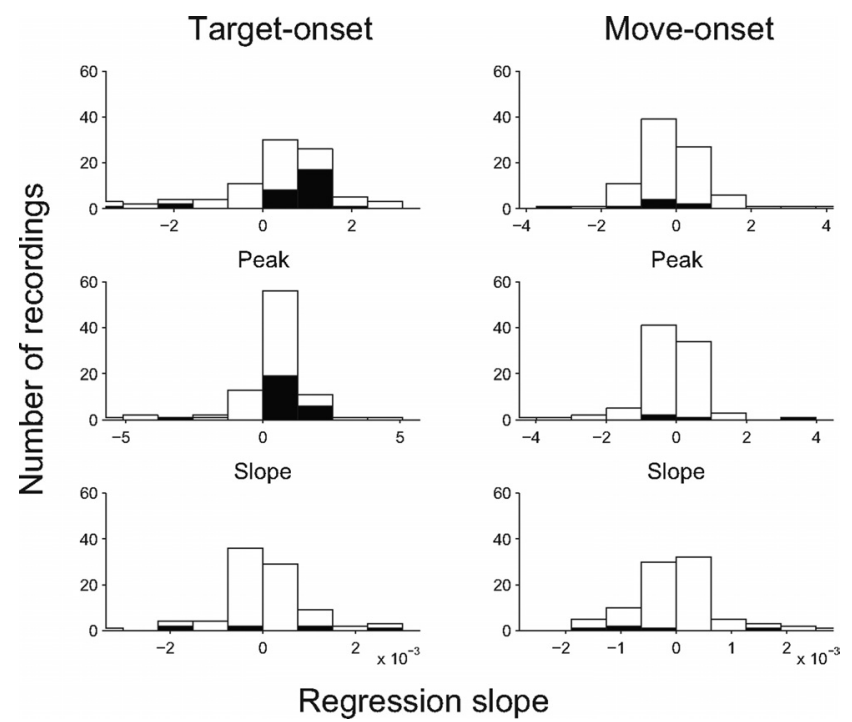

Figure 11. Distribution of regression coefficients describing the relationship of multiunit activity to reaction time across all recording sites. The distribution of regression coefficients between the timing of the reaction time and the timing of the activity onset (top), the timing of the peak activity (middle), and slope (bottom) when aligned on target presentation (left) and on arm movement initiation (right) is shown. Recordings with significant regression values are shown in black.

Table 2. Number of onset, peak, and slope of multiunit activity in SMA that were significantly correlated with arm movement reaction time

\begin{tabular}{ccrr}
\hline & \multicolumn{3}{l}{ SMA (all =94) } \\
\cline { 2 - 4 } & \# Sig & Mean (all) & \multicolumn{1}{l}{ Mean (sig) } \\
\hline Onset & & \\
$\quad$ Targ & $29(30.9 \%)$ & $0.293(p=0.034)$ & $0.522(p=0.021)$ \\
$\quad$ Move & $8(8.5 \%)$ & $-0.06(p=0.583)$ & $-0.745(p=0.095)$ \\
Peak & & & \\
$\quad$ Targ & $27(28.7 \%)$ & $0.424(p=0.007)$ & $0.791(p<0.001)$ \\
$\quad$ Move & $4(4.3 \%)$ & $-0.173(p=0.10)$ & $0.839(p=0.475)$ \\
Slope & & & \\
Targ & $7(7.4 \%)$ & $6.4 \times 10^{-5}(p=0.526)$ & $-2.4 \times 10^{-4}(p=0.728)$ \\
Move & $5(5.3 \%)$ & $-1.0 \times 10^{-5}(p=0.904)$ & $-5.3 \times 10^{-4}(p=0.352)$ \\
\hline & & &
\end{tabular}

trials (lower panels), the multiunit activity shown in Figure $14 \mathrm{~A}$ discharged significantly less in the target onset $(I=-0.274, p=$ $0.008)$ and movement $(I=-0.158, p=0.038)$ periods, but showed no significant change of activity during Ca-Go trials (upper panels). We also found cases in which the activity is different following both successful and unsuccessful responses to stop signals. For example, the multiunit activity shown in Figure $14 B$ showed an increase in firing rate during the baseline $(I=0.276$, $p=0.029)$ and movement $(I=0.163, p=0.028)$ periods in $\mathrm{Ca}-\mathrm{Go}$ trials, and a decrease in firing rate during the target $(I=$ $0.247, p=0.026)$ and movement $(I=-0.176, p=0.056)$ periods in E-Go trials.

As these examples show, the effects of trial history on multiunit activity varied across individual recordings. Therefore, we compared all recordings from each cortical area and monkey in the three temporal periods (Table 3; supplemental Fig. 3, available at www.jneurosci.org as supplemental material). In most cases, the activity was not significantly more likely to be higher (positive index) or lower (negative index) following a stop-signal trial. Likewise, significant activity changes were not significantly more likely to occur after canceled than noncanceled trials $\left(\chi^{2}\right.$ tests, $p>0.05)$. The only exception was multiunit activity recorded in the SMA of monkey B, which was more likely to be decreased than to be increased ( $\chi^{2}$ test, $p<0.01$ ), and more likely to be modulated following a noncanceled than a canceled trial $\left(\chi^{2}\right.$ test, $\left.p<0.05\right)$ (Table 3$)$.

\section{Single-unit activity is related to reaction time and reflects trial history}

So far, we have investigated the role of SMA in proactive control by analyzing measures of population activity: LFP and multiunit activity. Next, we determined whether we could find a role for these cortical areas in the preparatory regulation of motor readiness at the level of single-unit activity. To this end, we analyzed the 146 movement-related neurons in SMA that we recorded simultaneously with the LFP and multiunit activity (Scangos and Stuphorn, 2010). A multiple linear regression was performed to examine the relationship of single-unit movement-related activity to different movement parameters (Table 4). Thus, activity of 
Monkey B

A $\quad$ Ca-Go

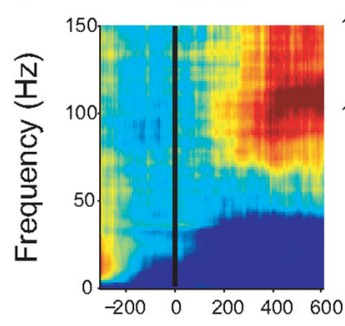

$\mathrm{B}$

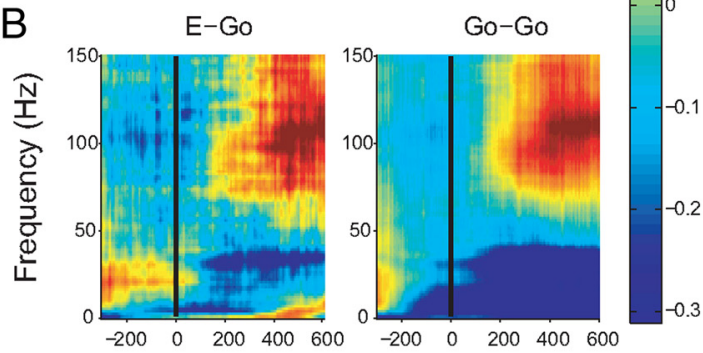

Time from target onset (ms)

C
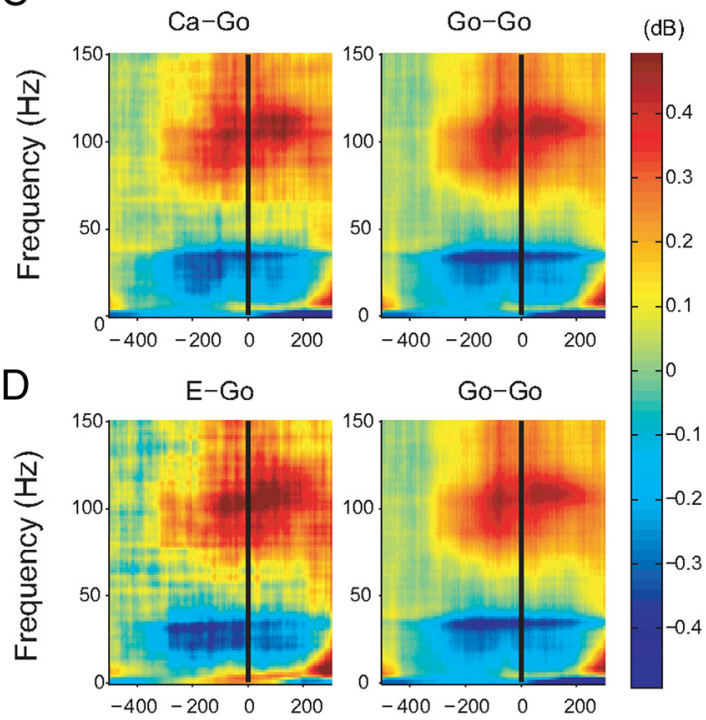

Time from movement onset (ms)

Figure 12. Effects of trial history on LFP power in the SMA of monkey B. Comparison was performed between three groups of no-stop-signal trials: those that followed another canceled trial ( $(\mathrm{C}-\mathrm{GO})$, those that followed a not-canceled error trial (E-Go), and those that followed a go trial (Go-Go). In the upper two rows $(\boldsymbol{A}, \boldsymbol{B})$, the time-frequency maps were aligned on target onset. $\boldsymbol{A}$, Comparison between $\mathrm{Ca}_{\mathrm{a}} \mathrm{G} 0$ and $\mathrm{Go}-\mathrm{Go}$ trials. The left panel shows grand average of time-frequency map of LFP power during $\mathrm{Ca}_{-} \mathrm{G} 0$, and the middle panel shows that during $\mathrm{G}_{0}-\mathrm{G}_{0}$ trials. The significant differences between them are shown in the right panel. $B$, Comparison between $\mathrm{E}-\mathrm{G} 0$ and $\mathrm{G} 0$-Go trials. In the lower two rows $(\boldsymbol{C}, \boldsymbol{D})$, the time-frequency maps were aligned on movement onset. C, Comparison between Ca-Go and Go-Go trials. D, Comparison between E-Go and Go-Go trials.

a large percentage of movement-related neurons in SMA was modified by response time $(40 \%, 57 / 146)$ or average velocity (52\%, 75/146).

We tested whether SMA neurons reflected trial history by comparing the level of activity on no-stop-signal trials following other no-stop-signal trials (Go-Go trials) with the level of activity on no-stop-signal trials following trials at which a stop signal was presented (Stop-Go trials). In the SMA, 16\% of movementrelated cells showed higher activity on Go-Go trials than Stop-Go trials, and $25 \%$ of neurons showed higher activity on Stop-Go trials than on Go-Go trials. Thus, a substantial number of movement-related cells in the pre-SMA (41\%) show significant changes in activity based on the previous trial type.

Figure 15 shows two SMA cells, whose activity was modified by response time. The recorded trials were split into five groups by response time, similar to the analysis of the multiunit activity. As in the case of multiunit activity, the activity increase of the single units also tended to be time locked to movement onset. Changes in reaction time were accompanied by shifts in the onset of the activity increase. SMA neurons do not directly control the initiation of arm movements (Scangos and Stuphorn, 2010). However, response time and movement velocity can both be interpreted as related to the level of excitability in the primary motor cortex (M1). Thus, the relationship of single units to reaction time further supports the idea that movement-related activity in SMA participates in the regulation of motor readiness.

\section{Changes in LFP power predict arm movement cancelation}

Up to this point, our investigations were exclusively with regard to the role of SMA in proactive control of arm movement generation. However, success in the stopsignal task obviously also requires the ability to cancel the ongoing movement preparation when a stop signal is given. This inhibitory control of the motor process is an example of reactive control in response to a sudden external cue. We reported previously that a small number of individual neurons in pre-SMA and SMA carried signals sufficient for inhibitory control of actions (Scangos and Stuphorn, 2010). Here, we tested whether the LFP activity in the SMA carried similar reactive inhibitory control signals. In this analysis, we included all SMA recordings with 10 or more canceled stopsignal trials.

In the stop-signal paradigm, the neuronal signal must fulfill two criteria to play a causally sufficient role in the control of action. First, the activity must discharge differently when a movement is initiated versus when it is canceled. Second, the activity difference in response to the stop signal must occur before the SSRT, which marks the end of the inhibitory process. Otherwise, it is too late to causally influence saccade initiation or inhibition.

We analyzed activity on canceled trials separately for each movement direction and SSD by comparing it with activity on latency-matched no-stop-signal trials, that is, the no-stop-signal trials with reaction time greater than the combined duration of the SSD plus SSRT. On these trials, the GO process was slow enough that if the stop signal had occurred, the GO process would have been interrupted by the faster STOP process. By ad- 
justing the reaction time distribution, we ensured that the state of motor readiness was similar, so that differences in LFP were not attributable to differences in proactive control. We tested for significant activity differences in the two types of trials by applying a permutation test to the power distribution in each pair of pixels in the two time-frequency maps beginning at $70 \mathrm{~ms}$ before SSRT and ending $150 \mathrm{~ms}$ after the SSRT. We downsampled the time-frequency maps with a $6 \mathrm{~ms}$ and 1.4 $\mathrm{Hz}$ resolution. The result was corrected for multiple comparisons using the FDR method.

Figure 16 shows the comparison of LFP power in a representative SMA recording. The time-frequency maps are aligned on target onset (first vertical line) and represent the activity for one specific SSD (second line). The SSRT is indicated by the third vertical line. For each SSD comparison, the upper time-frequency map shows activity during latency-matched no-stop-signal trials, while the lower map shows activity during canceled trials. For comparison, the simultaneously recorded multiunit activity is indicated by the thick black line overlaying the time-frequency maps. The smaller time-frequency map in the bottom row shows the significant differences in LFP power between the two conditions. Note that the map is shifted backward for the second SSD comparison, because the SSD is longer.

The comparison for each SSD showed that there were significant differences in LFP power in a number of frequency bands, which correlated with the successful cancelation of the arm movement. In the low-frequency band $(5-20 \mathrm{~Hz})$, especially for planned ipsilateral movements (Fig. 16A), there was an increase of activity right after the stop signal that lasts long after SSRT. Within the high gamma band $(130-140 \mathrm{~Hz})$, especially for planned contralateral movements (Fig. 16B), there was an increase of power immediately after the stop signal and before the SSRT. In addition, in all comparisons there was a complicated mixture of decreased and increased LFP power throughout the time-frequency map. Importantly, the modulation of LFP power in both recordings clearly started before the SSRT. This indicates that the neuronal processes that underlie the changes in LFP power in the respective parts of pre-SMA and SMA were sufficient to reactively control the cancelation of the arm movement.

A large number of LFP recordings in both SMA and pre-SMA showed such reactive control activity. We pooled the results of the different SSDs separately for movements in both directions, by averaging over the SSD-specific time-frequency maps of significant differences. In the SMA, 87\% (78/90) of recordings showed significant changes of LFP power in the beta band that started before the SSRT. Of these recordings, 60\% (47/78) showed significantly higher activity and 40\% (31/78) showed sig-
Monkey E
Go-Go
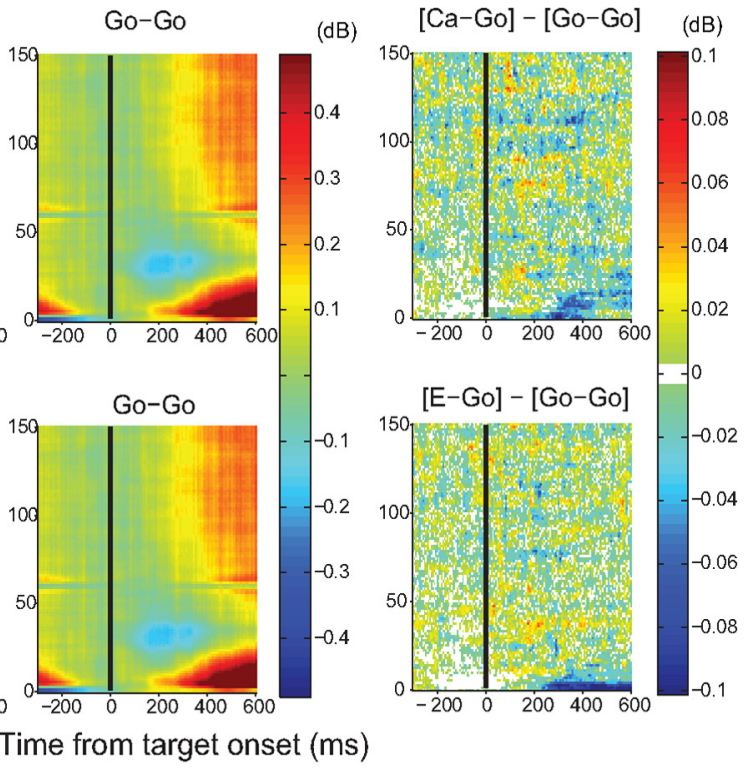

Time from target onset (ms)

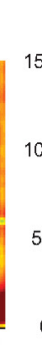

Go-Go

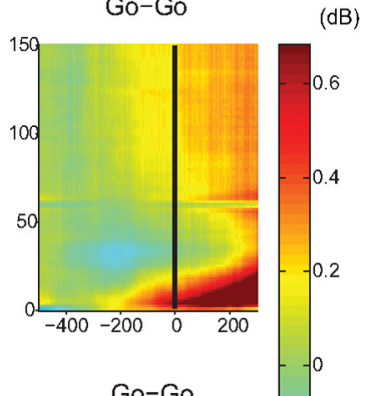

(dB)
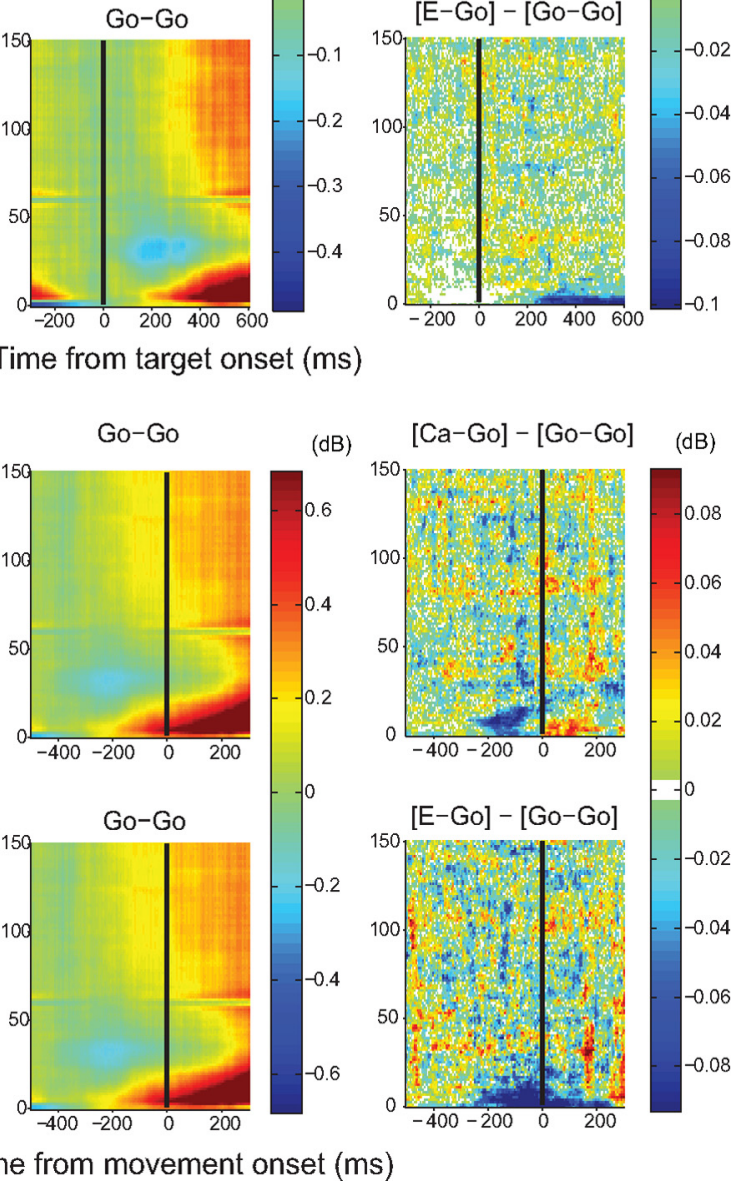

Time from movement onset (ms)

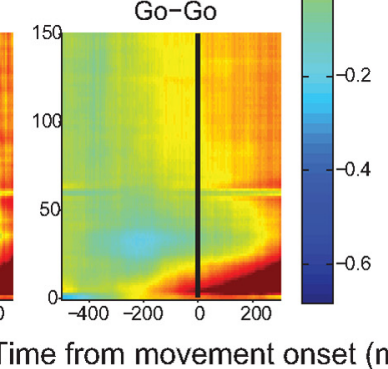

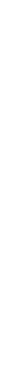

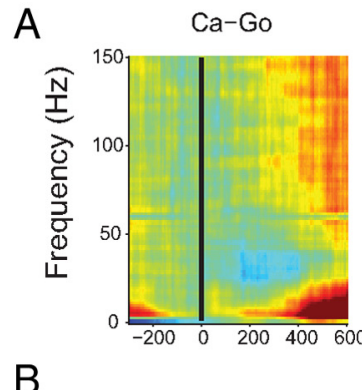

$\mathrm{Ca}-\mathrm{Go}$
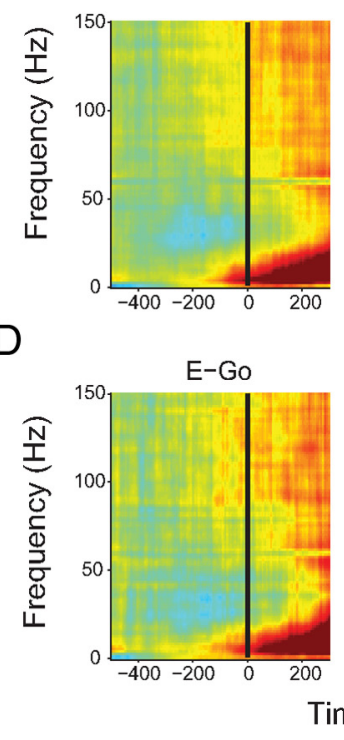

Figure 13. Effects of trial history on LFP power in the SMA of monkey E. Conventions are as in Figure 12.

nificantly lower activity. The activity differences in high gamma band represented a more complicated mixture of increases and decreases as described in the representative recording. A strong increase of gamma band activity generally occurred after the SSRT. However, in 23\% (21/90) of SMA recordings, there was a significant increase of high gamma activity before SSRT. This percentage is generally consistent with the results of single-unit analysis (Scangos and Stuphorn, 2010).

In the individual recordings, there was some variance in the specific time-frequency regions that did show significant increase or decreases of LFP power. We therefore combined all difference maps from each monkey into a grand average to determine a general trend across recordings. We aligned the timefrequency maps of significant differences in LFP power of each recording to SSRT. The grand average covers the time period beginning at $70 \mathrm{~ms}$ before the SSRT and $150 \mathrm{~ms}$ after the SSRT. Figure $17 \mathrm{~A}$ shows the comparison for the SMA recordings in 


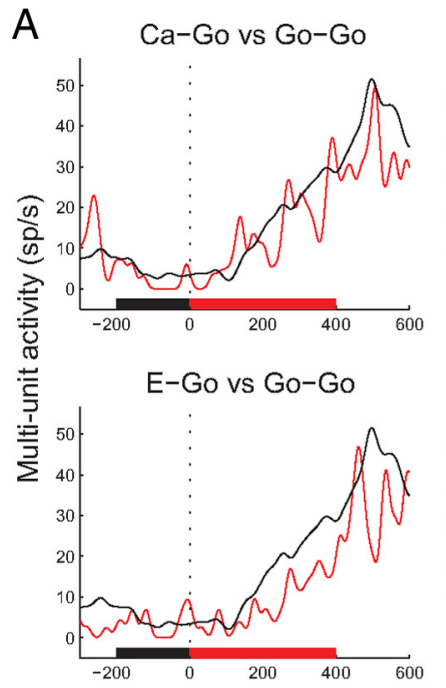

Time from Target Onset (ms) Time from Movement Onset (ms)
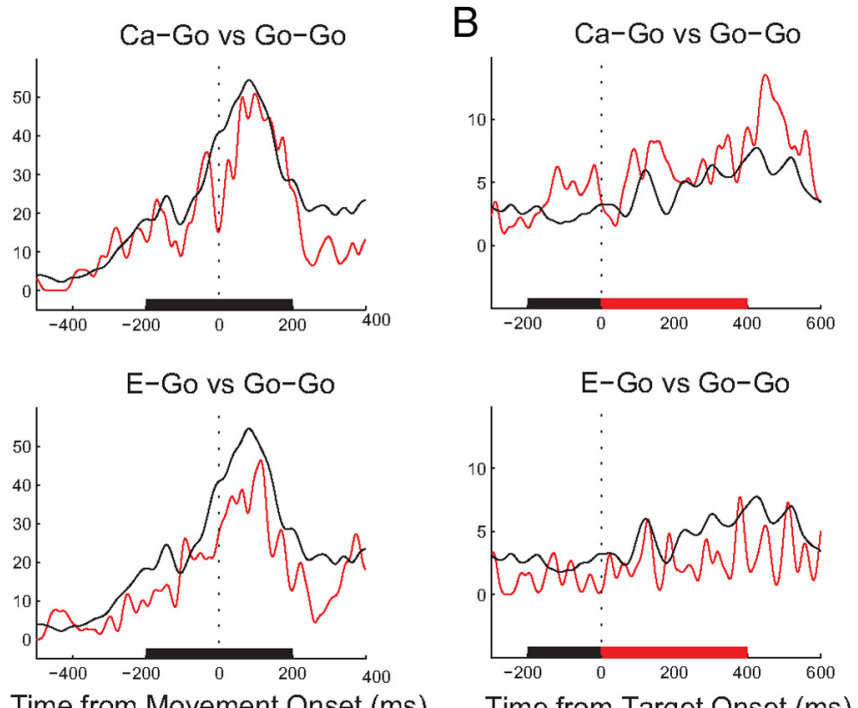

Time from Target Onset (ms)

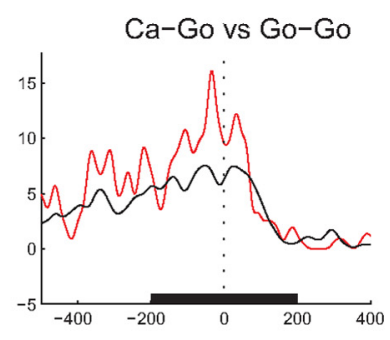

E-Go vs Go-Go

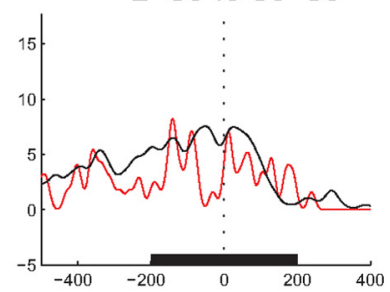

Time from Movement Onset (ms)

Figure 14. Effects of trial history on multiunit activity for two SMA recording sites. The panels in $\boldsymbol{A}$ and $\boldsymbol{B}$ show representative multiunit recordings from two different locations in the SMA of monkey B. The upper panels show the multiunit activity on Ca-Go trials (red) and on Go-Go trials (black) aligned on target (left) and movement (right) onset. The lower panels show the comparison between E-Go (red) and (black) Go-Go trials. Discharge rate was measured in three intervals indicated by the red and black lines and was tested for significant differences using a bootstrapping test.

Table 3. Proportion of significant sequential effect indices in the different cortical areas and monkeys

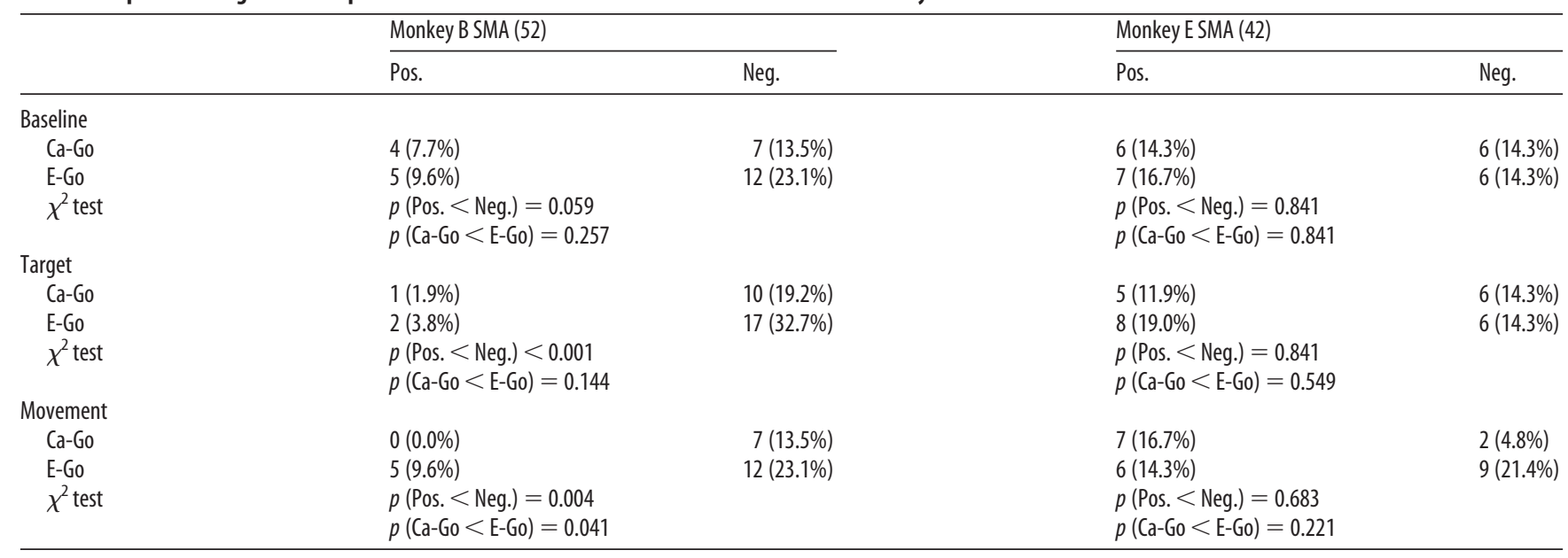

Table 4. Numbers of cells with activity modified by movement parameters

\begin{tabular}{lll}
\hline & Number of cells & Percent of movement related cells \\
\hline Velocity & 75 & $52 \%$ \\
Acceleration & 56 & $39 \%$ \\
Reaction Time & 57 & $40 \%$ \\
Direction & 94 & $70 \%$ \\
Length & 39 & $30 \%$ \\
\hline
\end{tabular}

monkey B. In addition to the map of significant differences (right panel; "Can-Go"), we also show the average LFP power map for canceled trials (middle panel; "Can"), and for the equivalent time periods in no-stop-signal trials (left panel; "Go"). The average LFP power showed differences in power in two frequency bands. The first one was the beta band $(10-50 \mathrm{~Hz})$. The LFP power in this band was reduced when an arm movement was executed. This reduction in power was less pronounced when the arm movement was canceled. The grand average of significant differences between these two groups therefore shows a relative increase of LFP power that started before SSRT. The second band that showed differences was within the high gamma range $(110-125 \mathrm{~Hz})$. For both ipsilateral and contralateral planned movements, there was a dramatic increase in activity starting $\sim 25 \mathrm{~ms}$ after SSRT. In addition, there was a decrease in activity before SSRT, but only for ipsilateral planned movement (upper row). The recordings in the SMA of monkey E (Fig. 17B) showed more diffuse effects across a wide band of frequencies ranging from the beta to the high gamma band that started before SSRT.

Thus, LFP recordings indicate that the SMA contributes to reactive control of arm movements. This short-latency activation involved both LFP power in the low-frequency band, which indicates increased synaptic activation, and the highfrequency band, which indicates increased firing rates. This finding confirms an earlier report (Scangos and Stuphorn, 2010).

\section{Discussion}

Our behavioral data showed strong sequential effects of errors and successful cancelations on the reaction time of arm movements in the stop-signal task. Errors or an increased frequency of stop-signal trials lead to longer reaction times on subsequent 
trials. Fewer stop-signal trials lead to shorter reaction times. The reaction time reflects the level of responsiveness in the motor system. A less excitable state leads to longer reaction times, while a more excitable state leads to shorter reaction times. The sequential effects show that the state of responsiveness of the motor system is constantly adjusted by control signals that reset the balance of excitation and inhibition within the motor system.

We have recorded neuronal activity in the SMA in the form of LFP, multiunit, and single-unit spiking activity, which was significantly correlated with reaction time. In particular, in LFP activity this correlation started to become significant $\sim 200 \mathrm{~ms}$ before target onset. Neuronal activity on all different scales also reflected recent trial history, which suggests that it might underlie the sequential behavioral effects. These findings indicate that SMA exerts proactive control over the skeletomotor system (for a comparison with other classifications of executive control, see supplemental material, available at www.jneurosci.org). These results are similar to those in an oculomotor stop-signal task (Emeric et al., 2007; Stuphorn et al., 2010).

In addition, in most recordings we also found increased LFP power during successful cancelations. This increase in power occurred early enough to be causally responsible for the inhibition of the movement. There might be an interaction between proactive and reactive forms of control (Chikazoe et al., 2009). Proactive control enhances the chance of a correct cancelation in case of a stop signal. However, such interactions cannot account for the existence of inhibitory neurons in pre-SMA and SMA (Scangos and Stuphorn, 2010). While numerically small in our recording sample, these neurons do not merely signal the need for inhibitory control. Instead, they are actively involved in inhibitory control, as shown by the fact that they were differentially active when the monkey withheld the movement, but not when he was unable to do so. These results provide proof of existence that the SMA, in addition to proactive control, can also exert reactive control over behavior.

Thus, our experimental results suggest that the SMA controls motor behavior through two different systems that work on different time scales. A reactive control system can respond to sudden changes in task demands by suppressing an action that has become counterproductive. This fast system is complemented by a slower proactive control system, which uses long-term expectations about task conditions to set the level of responsiveness in the motor system.

This hypothesis is in good agreement with neuroimaging studies that demonstrate robust activation of the medial frontal cortex, in particular of the pre-SMA, SMA, supplementary eye field, and anterior cingulate cortex in the countermanding paradigm (Curtis et al., 2005; Aron and Poldrack, 2006; Li et al., 2006; Aron et al., 2007a; Toxopeus et al., 2007). Patients with lesions in the dorsomedial cortex show response inhibition deficits in the stop-signal task (Floden and Stuss, 2006) and in Go/No-go tasks (Picton et al., 2007). Many human neuroimaging studies localize the activity in the stop-signal task to pre-SMA, but this activation likely includes neighboring regions, such as SMA. The two areas have important differences in their connectivity. The pre-SMA, but not the SMA, is connected with the prefrontal cortex, while
B

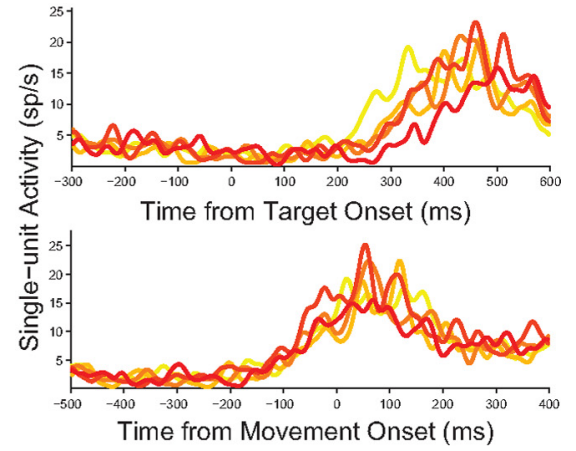

Figure 15. Single-unit neural activity modified by response time. The activity of two SMA neurons during movements toward their preferred direction in five reaction time groups was aligned on target presentation (upper row) and on arm movement initiation (lower row). Conventions are as in Figure 10. A, SMA neuron from monkey B. B, SMA neuron from monkey $\mathrm{E}$.

SMA, but not pre-SMA, is connected with M1 and the spinal cord. However, pre-SMA and SMA are also strongly interconnected, and the SMA clearly has a role in response inhibition (Sumner et al., 2007). The SMA might therefore provide a pathway, through which the pre-SMA influences the motor system. In humans, it has been shown that pre-SMA had a context-specific facilitatory effect on M1 activity (Mars et al., 2009). The latency of this effect is very short ( $6 \mathrm{~ms}$ ) and therefore unlikely to be dependent on subcortical pathways. Single-unit studies in monkeys do not show functional differences in the role of pre-SMA and SMA in the stop-signal task (Scangos and Stuphorn, 2010). In particular, the frequency of inhibitory neurons is the same in pre-SMA and SMA. It would be very interesting to combine future neuroimaging studies with DTI analysis to clearly demark the border between pre-SMA and SMA (Johansen-Berg et al., 2004).

In terms of computational reaction time models, a change in the responsiveness of the motor system translates into a shift of the distance to the threshold at which a response is initiated (Ratcliff, 1978; Luce, 1986; Reddi and Carpenter, 2000). A decrease of the threshold is equivalent to an increase of the baseline, and vice versa (Stuphorn and Schall, 2002; Bogacz et al., 2010). Such shifts can explain speed-accuracy tradeoffs (Uchida et al., 2006). The results of neurophysiological experiments fit such reaction time models very well. The firing rate of neurons in the oculomotor (Hanes and Schall, 1996) and skeletomotor system (Lecas et al., 1986) indeed exceeds a fixed threshold, when movements are initiated. There is also some evidence for changes in baseline activity in the oculomotor system. In the superior colliculus, neurons with visual and saccade-related activity increase their baseline firing rate with increasing probability that a saccade in their motor field is required (Basso and Wurtz, 1998; Dorris and Munoz, 1998) or is more rewarding (Isoda and Hikosaka, 2008).

We propose here that the dorsomedial frontal cortex, including the SMA, is the source of the proactive control signal that modulates the baseline motor activity. This hypothesis is supported by the fact that activity levels in and around the pre-SMA increased when response speed is emphasized during speed-accuracy tradeoff experiments (Forstmann et al., 2008; Ivanoff et al., 2008; van Veen et al., 2008).

We have shown in a previous study that movement-related neurons in pre-SMA and SMA do not carry signals sufficient to control the initiation of movements (Scangos and Stuphorn, 2010). Our findings here regarding the relationship of neuronal activity in the SMA with reaction time might be seen as contradicting these earlier findings. However, this is not the case. We 
A

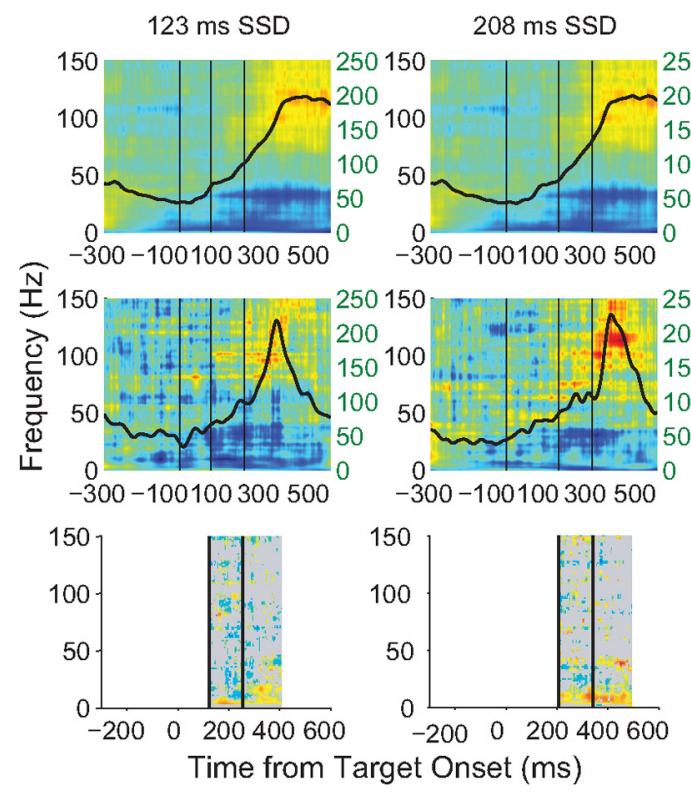

B
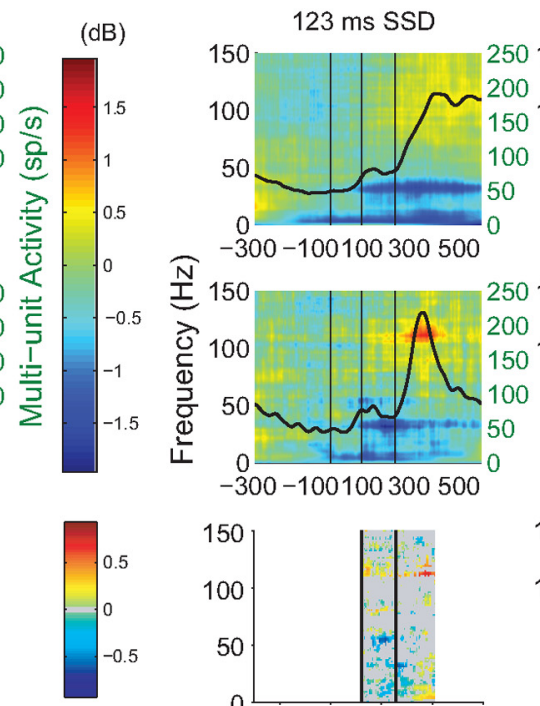

contra

$208 \mathrm{~ms}$ SSD

$(\mathrm{dB})$
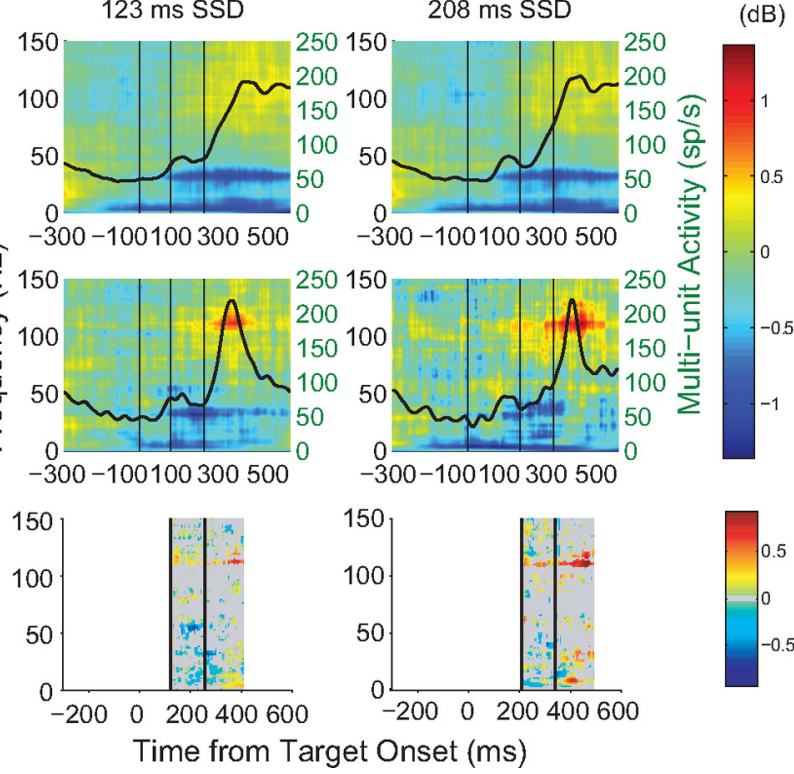

Figure 16. Changes in LFP power predict arm movement cancelation. A representative recording in the SMA of monkey B is shown for ipsilateral $(\boldsymbol{A})$ and contralateral ( $\boldsymbol{B})$ movements in the stop-signal task. The time-frequency maps represent the LFP activity aligned on target onset (first vertical line) for two different SSDs (second vertical line). The SSRT is indicated by the third vertical line. The panels in the upper row show the activity in latency-matched no-stop-signal trials, the middle panels show the activity during the canceled trials, and the lower panels show the significant difference between canceled and latency-matched no-stop-signal trials. The black line overlaying the time-frequency maps in the upper and middle panels indicates the simultaneously recorded multiunit activity. The gray background in the lower panels indicates the time period during which the LFP power was compared.

A

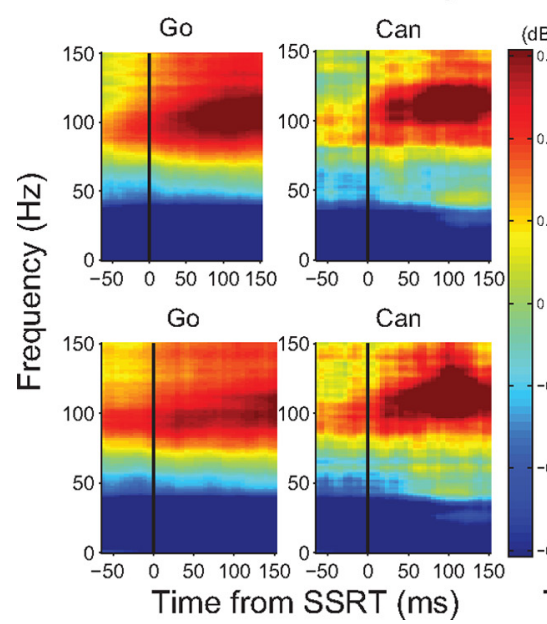

Monkey B

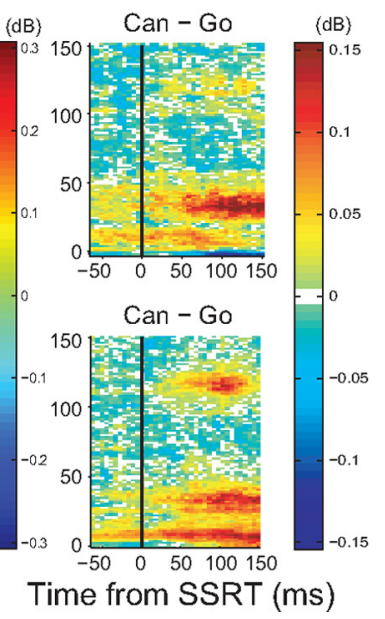

B

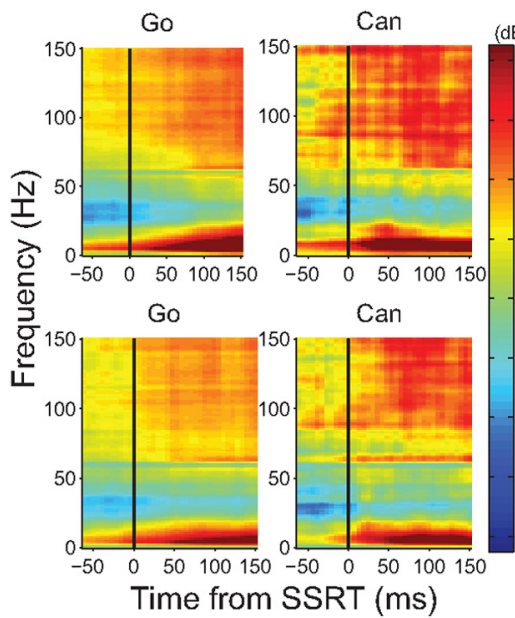

Monkey E

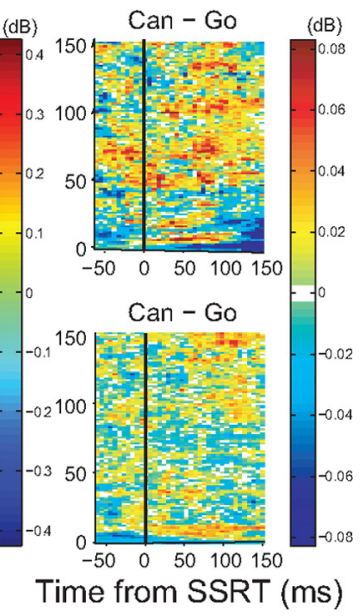

Figure 17. Grand average of comparison of LFP power between canceled and no-stop-signal trials. The grand average of the difference in LFP power was formed by taking the average of all the time-frequency maps aligned on the SSRT for each recording in the SMA of monkey $B(\boldsymbol{A})$ and of monkey $E(\boldsymbol{B})$. The panels on the left show the activity in latency-matched no-stop-signal trials ("Go"), the middle panels show the activity during canceled trials ("Can"), and the right panels show the significant difference between canceled and latency-matched no-stop-signal trials ("Can- $G 0^{\prime \prime}$ ). The panels in the upper row show ipsilateral movements, while the panels in the lower row show contralateral movements. For more details, see Results.

propose that SMA activity determines the response threshold, i.e., the amount of rise in motor activity that is necessary to initiate a movement. While the distance to the threshold clearly influences the average time at which it is exceeded, it is not sufficient to fully determine whether and when the threshold is actually exceeded. We propose that this process takes place in $M 1$, while SMA modulates this process by setting the urgency with which a movement is chosen and executed.

The neuronal mechanism underlying the influence of SMA on primary motor areas, such as M1, are not known. One possibility is direct or indirect corticocortical connections between SMA and M1. SMA projects to all other frontal motor cortical areas (Geyer et al., 2000). Another possibility is an indirect influence through the basal ganglia (Aron and Poldrack, 2006; Frank, 2006; Bogacz et al., 2010). Cortical input to the striatum and subthalamic nucleus (STN) could shift the balance of excitatory and inhibitory pathways within the basal ganglia, so that in a taskdependent manner the inhibitory control of the basal ganglia over the motor system could either be strengthened or lessened (Bogacz et al., 2010). Evidence supporting this possibility include the increased activation in the striatum with speed emphasis (Forstmann et al., 2008) and increased activation in the STN 
during successful cancelations (Aron and Poldrack, 2006; Aron et al., 2007a; Duann et al., 2009).

SMA most likely operates as node of a larger control network. Another area that has been found active in stop-signal tasks is the inferior frontal cortex (IFC) (Aron and Poldrack, 2006; Duann et al., 2009). A recent investigation of electrocorticographic (ECoG) brain recordings, similar to our analysis of the LFP recordings, showed more ECoG power in the beta band activity when human subjects successfully canceled a button press in a stop-signal task (Swann et al., 2009). The activity increase occurred early enough to be part of the cancelation process. However, the function of this neuronal activity is not known. It has been suggested that IFC participates directly in the movement inhibition (Aron and Poldrack, 2006). Alternatively, IFC is part of the ventral attention system, and could detect a salient, task-relevant event, such as a stop signal (Duann et al., 2009; Sharp et al., 2010). In any case, a connectivity study showed greater effective connectivity between pre-SMA and IFC during successful cancelations compared to unsuccessful ones (Duann et al., 2009).

In conclusion, our findings indicate that SMA, most likely in concert with a more extended neuronal network including preSMA, exerts proactive and reactive control over arm movements by adjusting the level of motor excitability.

\section{References}

Aron AR, Poldrack RA (2006) Cortical and subcortical contributions to stop signal response inhibition: role of the subthalamic nucleus. J Neurosci 26:2424-2433.

Aron AR, Behrens TE, Smith S, Frank MJ, Poldrack RA (2007a) Triangulating a cognitive control network using diffusion-weighted magnetic resonance imaging (MRI) and functional MRI. J Neurosci 27:3743-3752.

Aron AR, Durston S, Eagle DM, Logan GD, Stinear CM, Stuphorn V (2007b) Converging evidence for a fronto-basal-ganglia network for inhibitory control of action and cognition. J Neurosci 27:11860-11864.

Basso MA, Wurtz RH (1998) Modulation of neuronal activity in superior colliculus by changes in target probability. J Neurosci 18:7519-7534.

Benjamini Y, Yekutieli Y (2001) The control of the false discovery rate under dependency. Ann Stat 29:1165-1188.

Berens P, Keliris GA, Ecker AS, Logothetis NK, Tolias AS (2008) Comparing the feature selectivity of the gamma-band of the local field potential and the underlying spiking activity in primate visual cortex. Front Syst Neurosci 2:2.

Bogacz R, Wagenmakers EJ, Forstmann BU, Nieuwenhuis S (2010) The neural basis of the speed-accuracy tradeoff. Trends Neurosci 33:10-16.

Braver TS, Gray JR, Burgess GC (2007) Explaining the many varieties of working memory variation: dual mechanisms of cognitive control. In: Variation in working memory (Conway ARA, Jarrold C, Kane MJ, Miyake A, Towse JN, eds). Oxford: Oxford UP.

Bullmore ET, Suckling J, Overmeyer S, Rabe-Hesketh S, Taylor E, Brammer MJ (1999) Global, voxel, and cluster tests, by theory and permutation, for a difference between two groups of structural MR images of the brain. IEEE Trans Med Imaging 18:32-42.

Chikazoe J, Jimura K, Hirose S, Yamashita K, Miyashita Y, Konishi S (2009) Preparation to inhibit a response complements response inhibition during performance of a stop-signal task. J Neurosci 29:15870-15877.

Crone NE, Miglioretti DL, Gordon B, Lesser RP (1998a) Functional mapping of human sensorimotor cortex with electrocorticographic spectral analysis. II. Event-related synchronization in the gamma band. Brain 121:2301-2315.

Crone NE, Miglioretti DL, Gordon B, Sieracki JM, Wilson MT, Uematsu S, Lesser RP (1998b) Functional mapping of human sensorimotor cortex with electrocorticographic spectral analysis. I. Alpha and beta eventrelated desynchronization. Brain 121:2271-2299.

Curtis CE, Cole MW, Rao VY, D’Esposito M (2005) Canceling planned action: an FMRI study of countermanding saccades. Cereb Cortex 15:1281-1289.

Dorris MC, Munoz DP (1998) Saccadic probability influences motor preparation signals and time to saccadic initiation. J Neurosci 18:7015-7026.

Duann JR, Ide JS, Luo X, Li CS (2009) Functional connectivity delineates distinct roles of the inferior frontal cortex and presupplementary motor area in stop signal inhibition. J Neurosci 29:10171-10179.

Durka PJ, Zygierewicz J, Klekowicz H, Ginter J, Blinowska KJ (2004) On the statistical significance of event-related EEG desynchronization and synchronization in the time-frequency plane. IEEE Trans Biomed Eng 51:1167-1175.

Efron B, Tibshirani RJ (1993) An introduction to the bootstrap. Boca Raton, FL: Chapman and Hall/CRC.

Emeric EE, Brown JW, Boucher L, Carpenter RH, Hanes DP, Harris R, Logan GD, Mashru RN, Paré M, Pouget P, Stuphorn V, Taylor TL, Schall JD (2007) Influence of history on saccade countermanding performance in humans and macaque monkeys. Vision Res 47:35-49.

Floden D, Stuss DT (2006) Inhibitory control is slowed in patients with right superior medial frontal damage. J Cogn Neurosci 18:1843-1849.

Forstmann BU, Dutilh G, Brown S, Neumann J, von Cramon DY, Ridderinkhof KR, Wagenmakers EJ (2008) Striatum and pre-SMA facilitate decision-making under time pressure. Proc Natl Acad Sci U S A 105:17538-17542.

Frank MJ (2006) Hold your horses: a dynamic computational role for the subthalamic nucleus in decision making. Neural Netw 19:1120-1136.

Geyer S, Matelli M, Luppino G, Zilles K (2000) Functional neuroanatomy of the primate isocortical motor system. Anat Embryol (Berl) 202:443-474.

Hanes DP, Schall JD (1995) Countermanding saccades in macaque. Vis Neurosci 12:929-937.

Hanes DP, Schall JD (1996) Neural control of voluntary movement initiation. Science 274:427-430.

Hanes DP, Patterson WF 2nd, Schall JD (1998) Role of frontal eye fields in countermanding saccades: visual, movement, and fixation activity. J Neurophysiol 79:817-834.

Isoda M, Hikosaka O (2007) Switching from automatic to controlled action by monkey medial frontal cortex. Nat Neurosci 10:240-248.

Isoda M, Hikosaka O (2008) A neural correlate of motivational conflict in the superior colliculus of the macaque. J Neurophysiol 100:1332-1342.

Ito S, Stuphorn V, Brown JW, Schall JD (2003) Performance monitoring by the anterior cingulate cortex during saccade countermanding. Science 302:120-122.

Ivanoff J, Branning P, Marois R (2008) fMRI evidence for a dual process account of the speed-accuracy tradeoff in decision-making. PLoS One 3:e2635.

Johansen-Berg H, Behrens TE, Robson MD, Drobnjak I, Rushworth MF, Brady JM, Smith SM, Higham DJ, Matthews PM (2004) Changes in connectivity profiles define functionally distinct regions in human medial frontal cortex. Proc Natl Acad Sci U S A 101:13335-13340.

Lecas JC, Requin J, Anger C, Vitton N (1986) Changes in neuronal activity of the monkey precentral cortex during preparation for movement. J Neurophysiol 56:1680-1702.

Li CS, Huang C, Constable RT, Sinha R (2006) Imaging response inhibition in a stop-signal task: neural correlates independent of signal monitoring and post-response processing. J Neurosci 26:186-192.

Liu J, Newsome WT (2006) Local field potential in cortical area MT: stimulus tuning and behavioral correlations. J Neurosci 26:7779-7790.

Logan GD, Cowan WB (1984) On the ability to inhibit thought and action: a theory of an act of control. Psychol Rev 91:295-327.

Luce RD (1986) Response times and their role in inferring elementary mental organization. New York: Oxford UP.

Mallat SG, Zhang Z (1993) Matching pursuit with time-frequency dictionaries. IEEE Trans Signal Process 41:3397-3415.

Maris E, Oostenveld R (2007) Nonparametric statistical testing of EEG- and MEG-data. J Neurosci Methods 164:177-190.

Mars RB, Klein MC, Neubert FX, Olivier E, Buch ER, Boorman ED, Rushworth MF (2009) Short-latency influence of medial frontal cortex on primary motor cortex during action selection under conflict. J Neurosci 29:6926-6931.

Mitzdorf U (1985) Current source-density method and application in cat cerebral cortex: investigation of evoked potentials and EEG phenomena. Physiol Rev 65:37-100.

Paré M, Hanes DP (2003) Controlled movement processing: superior colliculus activity associated with countermanded saccades. J Neurosci 23:6480-6489.

Pfurtscheller G, Flotzinger D, Neuper C (1994) Differentiation between finger, toe and tongue movement in man based on $40 \mathrm{~Hz}$ EEG. Electroencephalogr Clin Neurophysiol 90:456-460. 
Pfurtscheller G, Graimann B, Huggins JE, Levine SP, Schuh LA (2003) Spatiotemporal patterns of beta desynchronization and gamma synchronization in corticographic data during self-paced movement. Clin Neurophysiol 114:1226-1236.

Picton TW, Stuss DT, Alexander MP, Shallice T, Binns MA, Gillingham S (2007) Effects of focal frontal lesions on response inhibition. Cereb Cortex 17:826-838.

Rabbitt PM (1966) Errors and error correction in choice-response tasks. J Exp Psychol 71:264-272.

Ratcliff R (1978) A theory of memory retrieval. Psychol Rev 83:59-108.

Ray S, Hsiao SS, Crone NE, Franaszczuk PJ, Niebur E (2008a) Effect of stimulus intensity on the spike-local field potential relationship in the secondary somatosensory cortex. J Neurosci 28:7334-7343.

Ray S, Crone NE, Niebur E, Franaszczuk PJ, Hsiao SS (2008b) Neural correlates of high-gamma oscillations $(60-200 \mathrm{~Hz})$ in macaque local field potentials and their potential implications in electrocorticography. J Neurosci 28:11526-11536.

Reddi BA, Carpenter RH (2000) The influence of urgency on decision time. Nat Neurosci 3:827-830.

Sayer RJ, Friedlander MJ, Redman SJ (1990) The time course and amplitude of EPSPs evoked at synapses between pairs of CA3/CA1 neurons in the hippocampal slice 10:826.

Scangos KW, Stuphorn V (2010) Medial frontal cortex motivates but does not control movement initiation in the countermanding task. J Neurosci 30:1968-1982.

Sharp DJ, Bonnelle V, De Boissezon X, Beckmann CF, James SG, Patel MC, Mehta MA (2010) Distinct frontal systems for response inhibition, attentional capture, and error processing. Proc Natl Acad Sci U S A 107:6106-6111.

Stuphorn V, Schall JD (2002) Neuronal control and monitoring of initiation of movements. Muscle Nerve 26:326-339.

Stuphorn V, Schall JD (2006) Executive control of countermanding saccades by the supplementary eye field. Nat Neurosci 9:925-931.
Stuphorn V, Taylor TL, Schall JD (2000) Performance monitoring by the supplementary eye field. Nature 408:857-860.

Stuphorn V, Brown JW, Schall JD (2010) Role of supplementary eye field in saccade initiation: executive not direct control. J Neurophysiol 103:801-816.

Sumner P, Nachev P, Morris P, Peters AM, Jackson SR, Kennard C, Husain M (2007) Human medial frontal cortex mediates unconscious inhibition of voluntary action. Neuron 54:697-711.

Swann N, Tandon N, Canolty R, Ellmore TM, McEvoy LK, Dreyer S, DiSano M, Aron AR (2009) Intracranial EEG reveals a time- and frequencyspecific role for the right inferior frontal gyrus and primary motor cortex in stopping initiated responses. J Neurosci 29:12675-12685.

Toxopeus CM, de Vries PM, de Jong BM, Johnson KA, George MS, Bohning DE, Walker J, Leenders KL (2007) Cerebral activation patterns related to initiation and inhibition of hand movement. Neuroreport 18:1557-1560.

Uchida N, Kepecs A, Mainen ZF (2006) Seeing at a glance, smelling in a whiff: rapid forms of perceptual decision making. Nat Rev Neurosci 7:485-491.

van Veen V, Krug MK, Carter CS (2008) The neural and computational basis of controlled speed-accuracy tradeoff during task performance. J Cogn Neurosci 20:1952-1965.

Verbruggen F, Logan GD (2009) Proactive adjustments of response strategies in the stop-signal paradigm. J Exp Psychol Hum Percept Perform 35:835-854.

Verbruggen F, Liefooghe B, Vandierendonck A (2004) The interaction between stop signal inhibition and distractor interference in the flanker and Stroop task. Acta Psychol (Amst) 116:21-37.

Verbruggen F, Liefooghe B, Vandierendonck A (2006) The effect of interference in the early processing stages on response inhibition in the stop signal task. Q J Exp Psychol (Colchester) 59:190-203.

Viswanathan A, Freeman RD (2007) Neurometabolic coupling in cerebral cortex reflects synaptic more than spiking activity. Nat Neurosci 10:13081312. 